\title{
Photochemical Nickel-Catalyzed C-H Arylation: Synthetic Scope and Mechanistic Investigations
}

\author{
Drew R. Heitz, John C. Tellis, and Gary A. Molander* \\ Roy and Diana Vagelos Laboratories, Department of Chemistry, \\ University of Pennsylvania, Philadelphia, \\ Pennsylvania 19104-6323 \\ *To whom correspondence should be addressed. E-mail: gmolandr@sas.upenn.edu
}

\section{Supplementary Material}

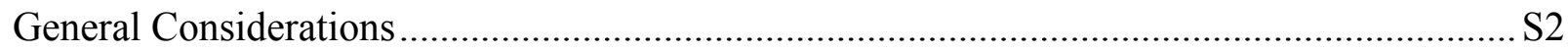

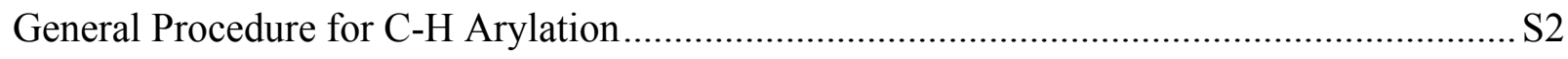

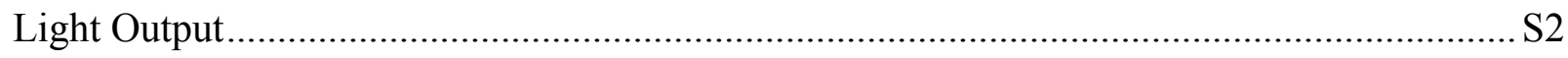

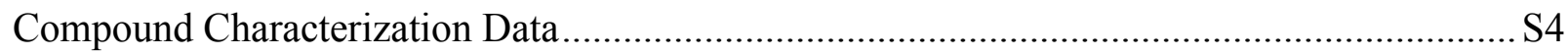

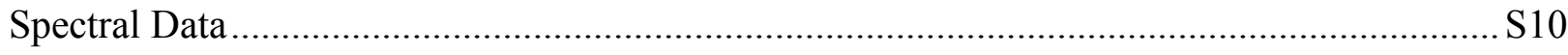

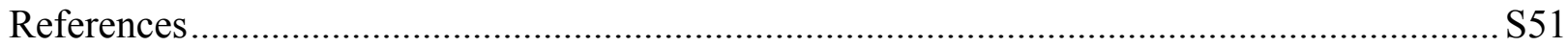




\section{General Considerations}

All reactions were carried out under an inert atmosphere of argon unless otherwise noted.

Solvents were purchased extra dry or distilled prior to use. $\mathrm{K}_{2} \mathrm{HPO}_{4}$ was used as received. The iridium photocatalyst, $\operatorname{Ir}\left[\mathrm{dFCF}_{3} \text { ppy }\right]_{2}(\mathrm{bpy}) \cdot \mathrm{PF}_{6}$, was synthesized from $\mathrm{IrCl}_{3} \cdot \mathrm{xH}_{2} \mathrm{O}$ according to our previously reported procedure. ${ }^{1} \mathrm{NiNO}_{3} \cdot 6 \mathrm{H}_{2} \mathrm{O}$ was purchased and used as received from a commercial source. Reactions were irradiated with two standard $26 \mathrm{~W}$ compact fluorescent light bulbs. Melting points $\left({ }^{\circ} \mathrm{C}\right)$ are uncorrected. Column chromatography was performed by Combiflash using RediSep Rf Gold Normal-Phase silica columns. ${ }^{1} \mathrm{H}(500 \mathrm{MHz})$ and ${ }^{13} \mathrm{C}(126$ $\mathrm{MHz}$ ) NMR chemical shifts are reported relative to internal TMS. HRMS spectra (ESI-TOF) were collected in $\mathrm{CH}_{2} \mathrm{Cl}_{2}$ or $\mathrm{MeCN}$.

\section{General Procedure for C-H Arylation}

4,4'-Di-tert-Butyl-2,2'-bipyridine (4.7 mg, $0.0175 \mathrm{mmol})$ and $\mathrm{NiNO}_{3} \cdot 6 \mathrm{H}_{2} \mathrm{O}(3.2 \mathrm{mg}, 0.0175$ mmol) were weighed into a $10 \mathrm{~mL}$ vial and were placed under argon. Then $1 \mathrm{~mL}$ of dry, degassed THF was added, and the mixture was heated at $50{ }^{\circ} \mathrm{C}$ until a pale green solution was obtained. For coupling reactions in THF, an additional $6 \mathrm{~mL}$ of THF was added ( $0.05 \mathrm{M}$ overall), followed by addition of the aryl bromide $(0.35 \mathrm{mmol}, 1$ equiv) (liquid aryl bromides were added with the solvent). $\operatorname{Ir}\left[\mathrm{dFCF}_{3} \mathrm{ppy}\right]_{2}(\mathrm{bpy}) \cdot \mathrm{PF}_{6} \quad(3.5 \mathrm{mg}, 2 \mathrm{~mol} \%, 0.02 \mathrm{mmol}), 4,4$ 'dimethoxybenzophenone $(21 \mathrm{mg}, 25 \mathrm{~mol} \%, 0.0875 \mathrm{mmol})$, and $\mathrm{K}_{2} \mathrm{HPO}_{4}(122 \mathrm{mg}, 2.0$ equiv, $0.7 \mathrm{mmol})$ were added sequentially. For reactions with other solvent-substrates, THF was removed, and $7 \mathrm{~mL}$ of distilled solvent $(0.05 \mathrm{M})$, aryl bromide $(0.35 \mathrm{mmol}, 1$ equiv) (liquid aryl bromides were added with the solvent), $\operatorname{Ir}\left[\mathrm{dFCF}_{3} \mathrm{ppy}\right]_{2}(\mathrm{bpy}) \cdot \mathrm{PF}_{6}(3.5 \mathrm{mg}, 2 \mathrm{~mol} \%, 0.02 \mathrm{mmol})$, 4,4'-dimethoxybenzophenone ( $21 \mathrm{mg}, 25 \mathrm{~mol} \mathrm{\%}, 0.0875 \mathrm{mmol}$ ), and $\mathrm{K}_{2} \mathrm{HPO}_{4}(122 \mathrm{mg}, 2.0$ equiv, $0.7 \mathrm{mmol}$ ) were added sequentially. The resulting mixtures was stirred approximately 4 $\mathrm{cm}$ away from two $26 \mathrm{~W}$ fluorescent light bulbs while a fan was blown across the reaction setup to maintain a temperature of $25{ }^{\circ} \mathrm{C}$. Reaction progress was monitored by HPLC, GCMS, or TLC. Upon consumption of aryl bromide, the crude reaction mixture was filtered through a cylindrical plug of Celite and rinsed with $\mathrm{CH}_{2} \mathrm{Cl}_{2}$ and EtOAc $(10-20 \mathrm{~mL})$. The filtrate was concentrated by rotary evaporation, and the residue was purified by column chromatography on silica gel, eluting with EtOAc and hexanes, to obtain products in pure form.
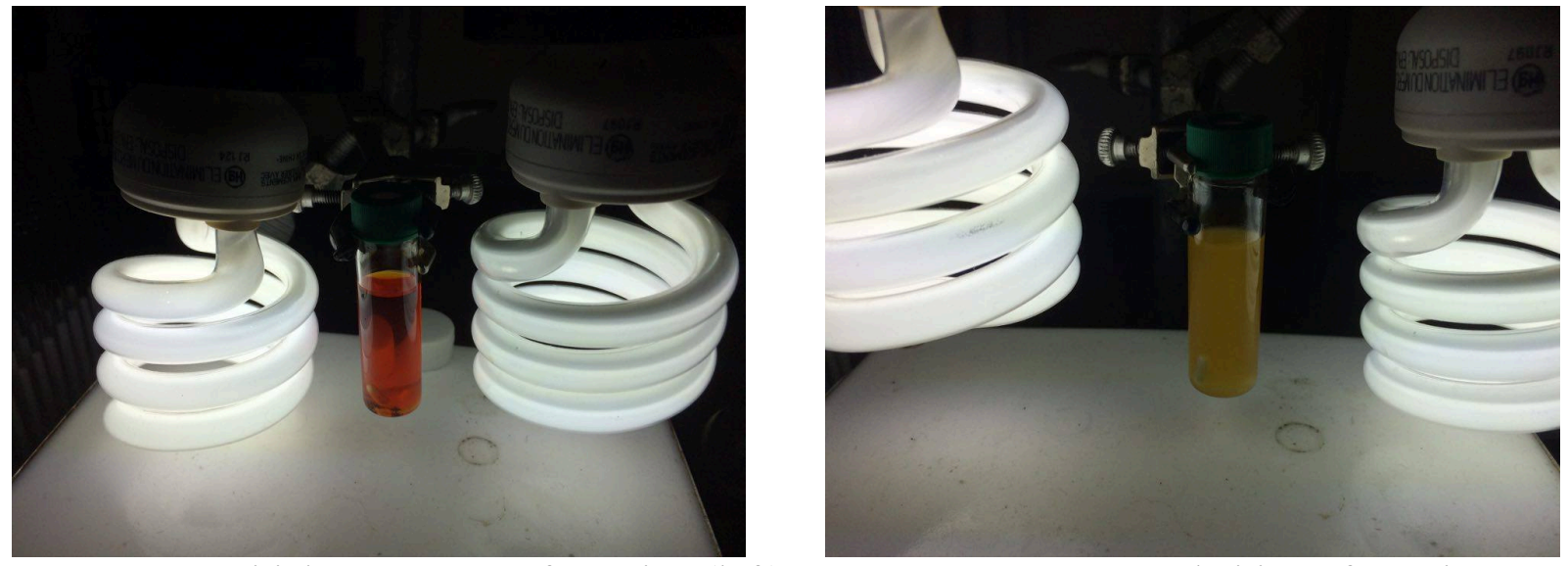

Figure S-1: Initial appearance of reaction (left). Appearance upon completition of reaction (right). 


\section{Light Output}

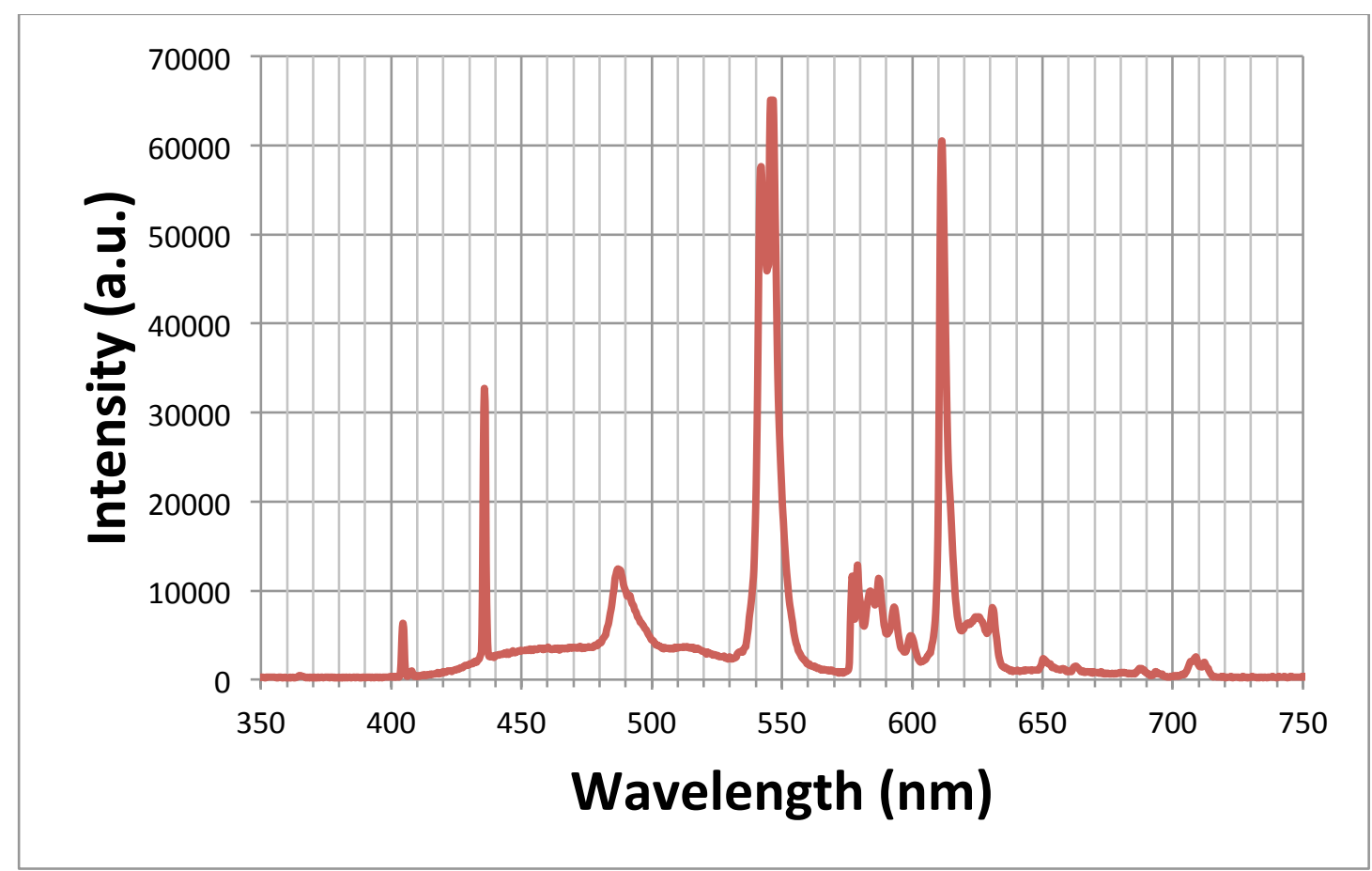

Figure S-2: Emission spectrum of $26 \mathrm{~W}$ CFL used for photocatalytic C-H arylation. 


\section{Compound Characterization Data}

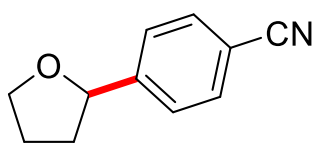

4-(Tetrahydrofuran-2-yl)benzonitrile (2): obtained as a colorless oil (70\%, $24 \mathrm{~h} ; 89 \%, 72 \mathrm{~h})$.

${ }^{1} \mathrm{H} \mathrm{NMR}\left(\mathrm{CDCl}_{3}, 500 \mathrm{MHz}\right): \delta=7.62(\mathrm{~d}, J=7.9 \mathrm{~Hz}, 2 \mathrm{H}), 7.43(\mathrm{~d}, J=7.7 \mathrm{~Hz}, 2 \mathrm{H}), 4.93(\mathrm{t}, J=$ $7.5 \mathrm{~Hz}, 1 \mathrm{H}), 4.09$ (q, $J=8.0 \mathrm{~Hz}, 1 \mathrm{H}), 3.96$ (q, $J=7.2 \mathrm{~Hz}, 1 \mathrm{H}), 2.37$ (m, 1H), $2.01(\mathrm{~m}, 2 \mathrm{H}), 1.74$ $(\mathrm{m}, 1 \mathrm{H})$.

${ }^{13} \mathrm{C}$ NMR (126 MHz, $\left.\mathrm{CDCl}_{3}\right): \delta=149.4,132.3,126.3,119.1,110.9,79.9,69.1,34.9,26.1$.

Characterization data matched that reported in the literature. ${ }^{2}$

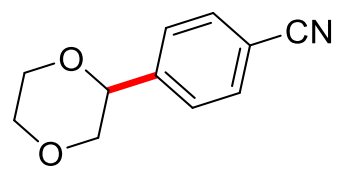

4-(1,4-Dioxan-2-yl)benzonitrile (5): obtained as a white semi-solid (22\%, $96 \mathrm{~h})$.

${ }^{1} \mathrm{H}$ NMR $\left(\mathrm{CDCl}_{3}, 500 \mathrm{MHz}\right): \delta=7.65(\mathrm{~d}, J=8.0 \mathrm{~Hz}, 2 \mathrm{H}), 7.47(\mathrm{~d}, J=8.0 \mathrm{~Hz}, 2 \mathrm{H}), 4.68(\mathrm{dd}, J$ $=10.2,2.8 \mathrm{~Hz}, 1 \mathrm{H}), 3.89,(\mathrm{~m}, 4 \mathrm{H}), 3.73(\mathrm{~m}, 1 \mathrm{H}), 3.38(\mathrm{~m}, 1 \mathrm{H})$.

${ }^{13} \mathrm{C} \mathrm{NMR}\left(126 \mathrm{MHz}, \mathrm{CDCl}_{3}\right) \delta=143.6,132.4,126.9,118.8,112.0,77.4,72.2,67.1,66.5$.

Characterization data matched that reported in the literature. ${ }^{3}$

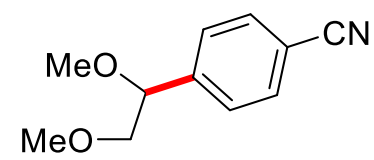

4-(1,2-Dimethoxyethyl)benzonitrile (6): obtained as an oil $(91 \%, 48 \mathrm{~h})$.

${ }^{1} \mathrm{H} \mathrm{NMR}\left(\mathrm{CDCl}_{3}, 500 \mathrm{MHz}\right): \delta=7.66(\mathrm{~d}, J=8.2 \mathrm{~Hz}, 2 \mathrm{H}), 7.46(\mathrm{~d}, J=8.2 \mathrm{~Hz}, 2 \mathrm{H}), 4.42(\mathrm{dd}, J$ $=7.2,4.0 \mathrm{~Hz}, 1 \mathrm{H}), 3.57,(\mathrm{dd}, J=10.3,7.3 \mathrm{~Hz}, 1 \mathrm{H}), 3.44(\mathrm{dd}, J=10.4,4.0 \mathrm{~Hz}, 1 \mathrm{H}), 3.37$ (s, $3 \mathrm{H})$, $3.31(\mathrm{~s}, 3 \mathrm{H})$.

${ }^{13} \mathrm{C} \mathrm{NMR}\left(126 \mathrm{MHz}, \mathrm{CDCl}_{3}\right) \delta=144.8,132.5,127.8,118.8,111.9,82.5,76.6,59.5,57.6$.

IR: $v=2931,2893,2827,2227,1609,1587,1513,1451,1352,1286,1192,1169,1098,1073$, $1034,869,838,584,569,549 \mathrm{~cm}^{-1}$.

HRMS: (ESI) m/z calc. for $\mathrm{C}_{11} \mathrm{H}_{14} \mathrm{NO}_{2}(\mathrm{M}+\mathrm{H})$ 192.1025, found 192.1019.

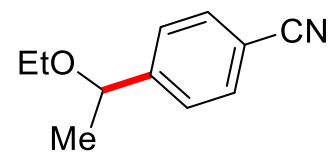

4-(1-Ethoxyethyl)benzonitrile (7): obtained as a colorless oil $(56 \%, 72 \mathrm{~h})$.

${ }^{1} \mathrm{H} \mathrm{NMR}\left(\mathrm{CDCl}_{3}, 500 \mathrm{MHz}\right): \delta=7.63(\mathrm{~d}, J=8.3 \mathrm{~Hz}, 2 \mathrm{H}), 7.42(\mathrm{~d}, J=8.2 \mathrm{~Hz}, 2 \mathrm{H}), 4.44(\mathrm{q}, J=$ $6.5 \mathrm{~Hz}, 1 \mathrm{H}), 3.36(\mathrm{~m}, 2 \mathrm{H}), 1.41(\mathrm{dd}, J=6.5,1.4 \mathrm{~Hz}, 3 \mathrm{H}), 1.20(\mathrm{td}, J=7.0,1.4 \mathrm{~Hz}, 3 \mathrm{H})$.

${ }^{13} \mathrm{C}$ NMR $\left(126 \mathrm{MHz}, \mathrm{CDCl}_{3}\right): \delta=150.1,132.5,126.8,119.1,111.2,77.3,64.6,24.2,15.5$. IR: $v=2977,2872,2229,1609,1371,1208,1100,1010,838,573 \mathrm{~cm}^{-1}$. 
HRMS: (ESI) m/z calc. for $\mathrm{C}_{11} \mathrm{H}_{14} \mathrm{NO}(\mathrm{M}+\mathrm{H})$ 176.1075, found 176.1061 .

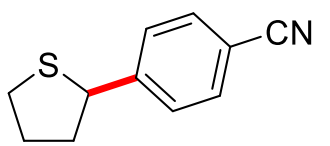

4-(Tetrahydrothiophen-2-yl)benzonitrile (9): obtained as a colorless oil (32\%, $72 \mathrm{~h})$.

${ }^{1} \mathrm{H} \mathrm{NMR}\left(\mathrm{CDCl}_{3}, 500 \mathrm{MHz}\right): \delta=7.59(\mathrm{~d}, J=8.3 \mathrm{~Hz}, 2 \mathrm{H}), 7.52(\mathrm{~d}, J=8.4 \mathrm{~Hz}, 2 \mathrm{H}), 4.52(\mathrm{dd}, J=$ 8.5, $6.4 \mathrm{~Hz}, 1 \mathrm{H}), 3.16(\mathrm{~m}, 1 \mathrm{H}), 3.03(\mathrm{~m}, 1 \mathrm{H}), 2.42(\mathrm{~m}, 1 \mathrm{H}), 2.26(\mathrm{~m}, 1 \mathrm{H}), 2.02(\mathrm{~m}, 1 \mathrm{H}), 1.90(\mathrm{~m}$, $1 \mathrm{H})$.

${ }^{13} \mathrm{C} \mathrm{NMR}\left(126 \mathrm{MHz}, \mathrm{CDCl}_{3}\right) \delta=149.1,132.4,128.6,119.0,110.9,52.4,40.6,33.8,31.2$.

Characterization data matched that reported in the literature. ${ }^{4}$

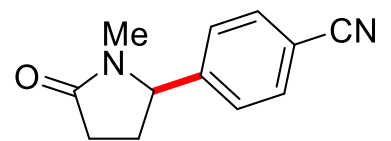

4-(1-Methyl-5-oxopyrrolidin-2-yl)benzonitrile (10): isolated as a light brown oil (81\%, $96 \mathrm{~h})$; mixture of secondary arylation product (major) with primary $N$-Me arylation isomer (8.3:1).

${ }^{1} \mathrm{H} \mathrm{NMR}\left(\mathrm{CDCl}_{3}, 500 \mathrm{MHz}\right): \delta=7.72(\mathrm{~d}, J=10 \mathrm{~Hz}, 2 \mathrm{H}), 7.35(\mathrm{~d}, J=10 \mathrm{~Hz}, 2 \mathrm{H}), 4.61(\mathrm{dd}, J=$ $5,5 \mathrm{~Hz}, 1 \mathrm{H}), 2.72(\mathrm{~s}, 3 \mathrm{H}), 2.60-2.47(\mathrm{~m}, 3 \mathrm{H}), 1.87-1.83(\mathrm{~m}, 1 \mathrm{H})$.

${ }^{13} \mathrm{C} \mathrm{NMR}\left(126 \mathrm{MHz}, \mathrm{CDCl}_{3}\right): \delta 175.5,146.7,133.0,127.1,118.4,112.0,64.1,29.8,28.4,28.2$.

Characterization data matched that reported in the literature. ${ }^{5}$

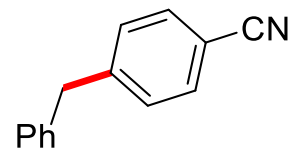

4-Benzylbenzonitrile (11): obtained as a white solid (87\%, $72 \mathrm{~h}), \mathrm{mp}=47-49{ }^{\circ} \mathrm{C}$.

${ }^{1} \mathrm{H}$ NMR $\left(\mathrm{CDCl}_{3}, 500 \mathrm{MHz}\right): \delta=7.57(\mathrm{~d}, J=8.0 \mathrm{~Hz}, 2 \mathrm{H}), 7.30(\mathrm{~m}, 5 \mathrm{H}), 7.17(\mathrm{~d}, J=7.4 \mathrm{~Hz}$, 2H), 4.09 (s, 2H).

${ }^{13} \mathrm{C} \mathrm{NMR}\left(126 \mathrm{MHz}, \mathrm{CDCl}_{3}\right) \delta=146.9,139.5,132.5,129.8,129.1,128.9,126.8,119.2,110.2$, 42.1.

Characterization data matched that reported in the literature. ${ }^{1}$

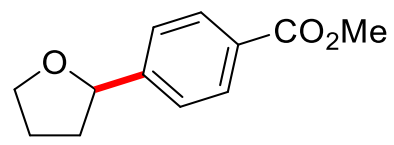

Methyl 4-(Tetrahydrofuran-2-yl)benzoate (13): obtained as a colorless oil (75\%, $48 \mathrm{~h})$.

${ }^{1} \mathrm{H}$ NMR $\left(\mathrm{CDCl}_{3}, 500 \mathrm{MHz}\right): \delta=8.00(\mathrm{~d}, J=8.3 \mathrm{~Hz}, 2 \mathrm{H}), 7.40(\mathrm{~d}, J=8.2 \mathrm{~Hz}, 2 \mathrm{H}), 4.94(\mathrm{t}, J=$ $7.2 \mathrm{~Hz}, 1 \mathrm{H}), 4.10(\mathrm{~m}, 1 \mathrm{H}), 3.96(\mathrm{q}, J=7.0 \mathrm{~Hz}, 1 \mathrm{H}), 3.91(\mathrm{~s}, 3 \mathrm{H}), 2.36(\mathrm{~m}, 1 \mathrm{H}), 2.01(\mathrm{~m}, 2 \mathrm{H})$, $1.78(\mathrm{~m}, 1 \mathrm{H})$.

${ }^{13} \mathrm{C} \mathrm{NMR}\left(126 \mathrm{MHz}, \mathrm{CDCl}_{3}\right): \delta=167.2,149.1,129.8,126.3,125.6,80.3,69.0,52.2,34.9,26.1$. 
Characterization data matched that reported in the literature. ${ }^{6}$

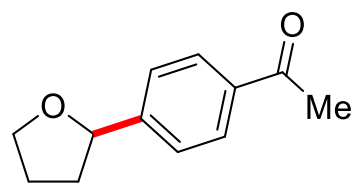

1-(4-(Tetrahydrofuran-2-yl)phenyl)ethan-1-one (14): product obtained with a trace of 4,4'dimethoxybenzophenone (xx:DMBP $=11.5: 1.0$ by ${ }^{1} \mathrm{H}$ NMR, 64\%, $48 \mathrm{~h}$ ).

${ }^{1} \mathrm{H} \mathrm{NMR}\left(\mathrm{CDCl}_{3}, 500 \mathrm{MHz}\right): \delta=7.92(\mathrm{~d}, J=8.3 \mathrm{~Hz}, 2 \mathrm{H}), 7.42(\mathrm{~d}, J=8.4 \mathrm{~Hz}, 2 \mathrm{H}), 4.95(\mathrm{t}, J=$ $7.2 \mathrm{~Hz}, 1 \mathrm{H}), 4.10(\mathrm{q}, J=6.9 \mathrm{~Hz}, 1 \mathrm{H}), 3.96(\mathrm{q}, J=8.0,1 \mathrm{H}) 2.59(\mathrm{~s}, 3 \mathrm{H}), 2.37(\mathrm{~m}, 1 \mathrm{H}), 2.01(\mathrm{~m}$, 2H), $1.77(\mathrm{~m}, 1 \mathrm{H})$.

${ }^{13} \mathrm{C} \mathrm{NMR}\left(126 \mathrm{MHz}, \mathrm{CDCl}_{3}\right): \delta=198.0,149.3,136.2,128.6,125.7,80.3,69.0,34.9,26.8,26.1$.

Characterization data matched that reported in the literature. ${ }^{2}$

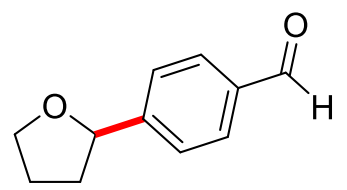

4-(Tetrahydrofuran-2-yl)benzaldehyde (15): using standard conditions, obtained as an inseparable mixture with 4,4'-dimethoxybenzophenone (71\%, $48 \mathrm{~h})$; in the absence of DMBP, obtained a clear oil $(57 \%, 72 \mathrm{~h})$.

${ }^{1} \mathrm{H} \mathrm{NMR}\left(\mathrm{CDCl}_{3}, 500 \mathrm{MHz}\right): \delta=10.00(\mathrm{~s}, 1 \mathrm{H}), 7.85(\mathrm{~d}, J=8.3 \mathrm{~Hz}, 2 \mathrm{H}), 7.50(\mathrm{~d}, J=7.9 \mathrm{~Hz}$, 2H), 4.97 (t, $J=7.2 \mathrm{~Hz}, 1 \mathrm{H}), 4.12(\mathrm{q}, 1 \mathrm{H}), 3.98(\mathrm{q}, 1 \mathrm{H}), 2.39(\mathrm{~m}, 1 \mathrm{H}), 2.02(\mathrm{~m}, 2 \mathrm{H}), 1.78(\mathrm{~m}$, $1 \mathrm{H})$.

${ }^{13} \mathrm{C} \mathrm{NMR}\left(126 \mathrm{MHz}, \mathrm{CDCl}_{3}\right): \delta=192.2,151.0,135.6,130.0,126.2,80.3,69.1,34.9,26.1$

IR: 2975, 2874, 1699, 1603, 1577, 1509, 1418, 1305, 1285, 1209, 1167, 1114, 1061, 1028, 927, $829,771 \mathrm{~cm}^{-1}$.

MS: (ESI) m/z calc. for $\mathrm{C}_{11} \mathrm{H}_{12} \mathrm{O}_{2}\left(\mathrm{M}^{+}\right)$176.0837, found 176.0827 .

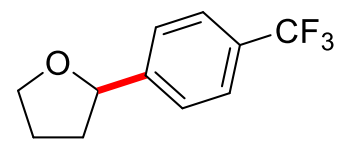

2-(4-(Trifluoromethyl)phenyl)tetrahydrofuran (16): using standard conditions, obtained a clear oil (72\% NMR yield, 63\% isolated yield, $72 \mathrm{~h})$; in the absence of DMBP, obtained a clear oil $(59 \%, 72 \mathrm{~h})$.

${ }^{1} \mathrm{H} \mathrm{NMR}\left(\mathrm{CDCl}_{3}, 500 \mathrm{MHz}\right): \delta=7.58(\mathrm{~d}, J=8.1 \mathrm{~Hz}, 2 \mathrm{H}), 7.44(\mathrm{~d}, J=8.0 \mathrm{~Hz}, 2 \mathrm{H}), 4.95(\mathrm{t}, J=$ $7.2 \mathrm{~Hz}, 1 \mathrm{H}), 4.11(\mathrm{dt}, J=8.4,6.8 \mathrm{~Hz}, 1 \mathrm{H}), 3.96(\mathrm{dt}, J=8.3,7.0 \mathrm{~Hz}, 1 \mathrm{H}), 2.37$ (dq, $J=13.2,6.7$ $\mathrm{Hz}, 1 \mathrm{H}), 2.01(\mathrm{~m}, 2 \mathrm{H}), 1.77(\mathrm{dq}, J=12.3,7.8 \mathrm{~Hz}, 1 \mathrm{H})$.

${ }^{13} \mathrm{C} \mathrm{NMR}\left(126 \mathrm{MHz}, \mathrm{CDCl}_{3}\right): \delta=147.9,129.6,125.9,125.4(\mathrm{q}, J=3.9 \mathrm{~Hz}), 123.3,80.1,69.0$, 34.9, 26.1 .

${ }^{19} \mathrm{~F} \mathrm{NMR}\left(\mathrm{CDCl}_{3}, 470.8 \mathrm{MHz}\right): \delta=-62.4$.

Characterization data matched that reported in the literature. ${ }^{3}$ 


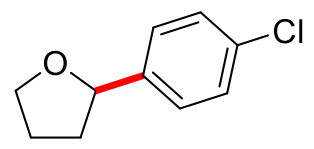

2-(4-Chlorophenyl)tetrahydrofuran (17): using standard conditions, obtained a clear oil (52\%, $72 \mathrm{~h})$; in the absence of DMBP, obtained a clear oil $(48 \%, 72 \mathrm{~h})$.

${ }^{1} \mathrm{H} \mathrm{NMR}\left(\mathrm{CDCl}_{3}, 500 \mathrm{MHz}\right): \delta=7.34-7.22(\mathrm{~m}, 4 \mathrm{H}), 4.86(\mathrm{t}, J=7.2 \mathrm{~Hz}, 1 \mathrm{H}), 4.08(\mathrm{dt}, J=8.4$, $6.8 \mathrm{~Hz}, 1 \mathrm{H}), 3.93(\mathrm{dt}, J=8.3,6.9 \mathrm{~Hz}, 1 \mathrm{H}), 2.32(\mathrm{~m}, 1 \mathrm{H}), 2.00(\mathrm{~m}, 2 \mathrm{H}), 1.75(\mathrm{~m}, 1 \mathrm{H})$.

${ }^{13} \mathrm{C} \mathrm{NMR}\left(126 \mathrm{MHz}, \mathrm{CDCl}_{3}\right): \delta=142.2,132.9,128.5,127.1,80.1,68.9,34.8,26.1$.

Characterization data matched that reported in the literature. ${ }^{3}$

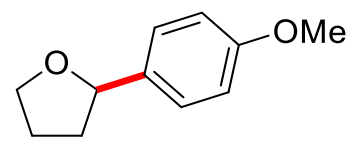

2-(4-Methoxyphenyl)tetrahydrofuran (18): obtained as a colorless oil (76\%, $72 \mathrm{~h})$.

${ }^{1} \mathrm{H} \mathrm{NMR}\left(\mathrm{CDCl}_{3}, 500 \mathrm{MHz}\right): \delta=7.26(\mathrm{~d}, J=8.6 \mathrm{~Hz}, 2 \mathrm{H}), 6.87(\mathrm{~d}, J=8.7 \mathrm{~Hz}, 2 \mathrm{H}), 4.83(\mathrm{t}, J=$ $7.2 \mathrm{~Hz}, 1 \mathrm{H}), 4.12-4.03(\mathrm{~m}, 1 \mathrm{H}), 3.91(\mathrm{td}, J=8.1,6.3 \mathrm{~Hz}, 1 \mathrm{H}), 3.80(\mathrm{~s}, 3 \mathrm{H}), 2.32-2.21(\mathrm{~m}$, $1 \mathrm{H}), 2.07-1.93(\mathrm{~m}, 2 \mathrm{H}), 1.79(\mathrm{~m}, 1 \mathrm{H})$.

${ }^{13} \mathrm{C} \mathrm{NMR}\left(126 \mathrm{MHz}, \mathrm{CDCl}_{3}\right): \delta=158.9,135.5,127.1,113.8,80.6,68.6,55.4,34.6,26.2$.

Characterization data matched that reported in the literature. ${ }^{3}$

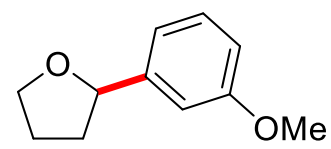

2-(3-Methoxyphenyl)tetrahydrofuran (19): obtained as a colorless oil $(62 \%, 72 \mathrm{~h})$.

${ }^{1} \mathrm{H}$ NMR $\left(\mathrm{CDCl}_{3}, 500 \mathrm{MHz}\right): \delta=7.24(\mathrm{~d}, J=8.1 \mathrm{~Hz}, 1 \mathrm{H}), 6.91(\mathrm{~d}, J=5.5 \mathrm{~Hz}, 2 \mathrm{H}), 6.79(\mathrm{dd}, J=$ $8.1,2.4 \mathrm{~Hz}, 1 \mathrm{H}), 4.88(\mathrm{t}, J=7.1 \mathrm{~Hz}, 1 \mathrm{H}), 4.09$ (q, $J=7.1 \mathrm{~Hz}, 1 \mathrm{H}), 3.93(\mathrm{q}, J=8.0 \mathrm{~Hz}, 1 \mathrm{H}), 3.81$ $(\mathrm{s}, 3 \mathrm{H}), 2.37-2.27(\mathrm{~m}, 1 \mathrm{H}), 2.06-1.94(\mathrm{~m}, 2 \mathrm{H}), 1.86-1.75(\mathrm{~m}, 1 \mathrm{H})$.

${ }^{13} \mathrm{C} \mathrm{NMR}\left(126 \mathrm{MHz}, \mathrm{CDCl}_{3}\right): \delta=159.8,145.4,129.4,118.1,112.7,111.2,80.6,68.8,55.4$, 34.7, 26.1.

Characterization data matched that reported in the literature. ${ }^{7}$

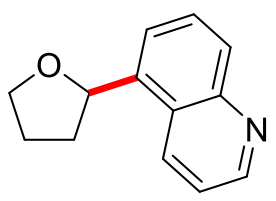

5-(Tetrahydrofuran-2-yl)quinoline (20): obtained as a pale yellow oil (54\%, $48 \mathrm{~h})$.

${ }^{1} \mathrm{H} \mathrm{NMR}\left(\mathrm{CDCl}_{3}, 500 \mathrm{MHz}\right): \delta=8.92(\mathrm{~d}, J=3.8 \mathrm{~Hz}, 1 \mathrm{H}), 8.37(\mathrm{~d}, J=8.5 \mathrm{~Hz}, 1 \mathrm{H}), 8.02(\mathrm{~d}, J=$ $9.2 \mathrm{~Hz}, 1 \mathrm{H}), 7.68(\mathrm{~d}, J=7.2 \mathrm{~Hz}, 2 \mathrm{H}), 7.41(\mathrm{dd}, J=8.5,4.1 \mathrm{~Hz}, 1 \mathrm{H}), 5.56(\mathrm{t}, J=7.1 \mathrm{~Hz}, 1 \mathrm{H})$, $4.22(\mathrm{q}, J=7.8,1 \mathrm{H}), 4.03(\mathrm{q}, J=7.5 \mathrm{~Hz}, 1 \mathrm{H}), 2.51(\mathrm{~m}, 1 \mathrm{H}), 2.08(\mathrm{~m}, 2 \mathrm{H}), 1.92(\mathrm{~m}, 1 \mathrm{H})$.

${ }^{13} \mathrm{C} \mathrm{NMR}\left(126 \mathrm{MHz}, \mathrm{CDCl}_{3}\right): \delta=150.0,148.7,139.7,132.2,129.2,129.0,125.8,122.8,120.8$, $77.8,68.9,33.9,26.1$. 
IR: $v=3063,2974,2869,1611,1595,1573,1500,1469,1372,1317,1150,1072,1048,1007$, 928, 843, 827, 802, 748, $558 \mathrm{~cm}^{-1}$.

HRMS: (ESI) m/z calc. for $\mathrm{C}_{14} \mathrm{H}_{14} \mathrm{NO}(\mathrm{M}+\mathrm{H}) 200.1075$, found 200.1039.

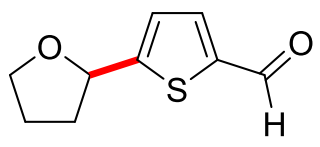

5-(Tetrahydrofuran-2-yl)thiophene-2-carbaldehyde (21): obtained as a pale yellow oil (43\%, $48 \mathrm{~h})$.

${ }^{1} \mathrm{H}$ NMR $\left(\mathrm{CDCl}_{3}, 500 \mathrm{MHz}\right): \delta=9.85(\mathrm{~s}, 1 \mathrm{H}), 7.64(\mathrm{~d}, J=3.8 \mathrm{~Hz}, 1 \mathrm{H}), 7.03(\mathrm{~d}, J=3.6 \mathrm{~Hz}, 1 \mathrm{H})$, $5.18(\mathrm{t}, J=6.6 \mathrm{~Hz}, 1 \mathrm{H}), 4.08(\mathrm{~m}, 1 \mathrm{H}), 3.93(\mathrm{~m}, 1 \mathrm{H}), 2.39(\mathrm{~m}, 1 \mathrm{H}), 2.11-1.87(\mathrm{~m}, 3 \mathrm{H})$.

${ }^{13} \mathrm{C} \mathrm{NMR}\left(126 \mathrm{MHz} \mathrm{CDCl}_{3}\right): \delta=183.1,159.4,142.3,136.8,124.3,77.4,69.0,35.0,26.0$.

IR: $v=2926,1710,1666,1528,1461,1228,1202,1057,923,815,753,670 \mathrm{~cm}^{-1}$.

HRMS: (ESI) m/z calc. for $\mathrm{C}_{9} \mathrm{H}_{10} \mathrm{O}_{2} \mathrm{~S}\left(\mathrm{M}^{+}\right)$182.0402, found 182.0398 .

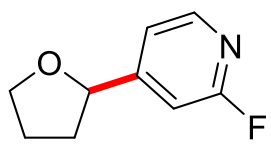

2-Fluoro-4-(tetrahydrofuran-2-yl)pyridine (22): obtained as a colorless oil (86\%, $72 \mathrm{~h})$.

${ }^{1} \mathrm{H} \mathrm{NMR}\left(\mathrm{CDCl}_{3}, 500 \mathrm{MHz}\right): \delta=8.14(\mathrm{~d}, J=5.2 \mathrm{~Hz}, 1 \mathrm{H}), 7.10(\mathrm{~d}, J=5.1 \mathrm{~Hz}, 1 \mathrm{H}), 6.91(\mathrm{~s}, 1 \mathrm{H})$, $4.92(\mathrm{t}, J=7.2 \mathrm{~Hz}, 1 \mathrm{H}), 4.08(\mathrm{q}, J=7.3 \mathrm{~Hz}, 1 \mathrm{H}), 3.96(\mathrm{q}, J=7.5 \mathrm{~Hz}, 1 \mathrm{H}), 2.40(\mathrm{~m}, 1 \mathrm{H}), 1.99(\mathrm{~m}$, $2 \mathrm{H}), 1.81(\mathrm{~m}, 1 \mathrm{H})$.

${ }^{13} \mathrm{C} \mathrm{NMR}\left(126 \mathrm{MHz} \mathrm{CDCl}_{3}\right): \delta=147.7,147.6,118.4,106.3,106.0,78.9,69.2,34.4,25.9$

${ }^{19} \mathrm{~F} \mathrm{NMR}\left(\mathrm{CDCl}_{3}, 470.8 \mathrm{MHz}\right): \delta=-68.2$.

IR: $v=2979,2874,1614,1568,1481,1405,1293,1274,1067,877,842 \mathrm{~cm}^{-1}$.

HRMS: (ESI) m/z calc. for $\mathrm{C}_{9} \mathrm{H}_{11} \mathrm{FNO}(\mathrm{M}+\mathrm{H}) 168.0825$, found 168.0822.

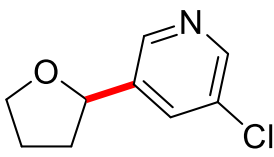

3-Chloro-5-(tetrahydrofuran-2-yl)pyridine (23): obtained as a colorless oil (62\%, $72 \mathrm{~h})$.

${ }^{1} \mathrm{H}$ NMR $\left(\mathrm{CDCl}_{3}, 500 \mathrm{MHz}\right): \delta=8.45(\mathrm{~d}, J=2.4 \mathrm{~Hz}, 1 \mathrm{H}), 8.42(\mathrm{~d}, J=1.8 \mathrm{~Hz}, 1 \mathrm{H}), 7.67(\mathrm{~s}, 1 \mathrm{H})$, $4.90(\mathrm{t}, J=7.2 \mathrm{~Hz}, 1 \mathrm{H}), 4.08(\mathrm{~m}, 1 \mathrm{H}), 3.94(\mathrm{~m}, 1 \mathrm{H}), 2.38(\mathrm{~m}, 1 \mathrm{H}), 2.02(\mathrm{~m}, 2 \mathrm{H}), 1.78(\mathrm{~m}, 1 \mathrm{H})$.

${ }^{13} \mathrm{C} \mathrm{NMR}\left(126 \mathrm{MHz}, \mathrm{CDCl}_{3}\right): \delta=147.6,145.5,140.6,133.2,132.1,77.8,69.0,34.7,26.1$.

IR: $v=2977,2872,1582,1559,1440,1421,1360,1298,1233,1102,1063,1022,925,880,704$ $\mathrm{cm}^{-1}$.

HRMS: (ESI) m/z calc. for $\mathrm{C}_{9} \mathrm{H}_{11} \mathrm{ClNO}(\mathrm{M}+\mathrm{H})$ 184.0524, found 184.0523.

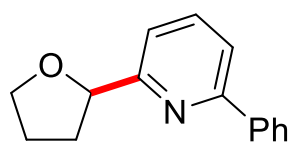


2-Phenyl-6-(tetrahydrofuran-2-yl)pyridine (24): obtained as a colorless oil (49\%, $72 \mathrm{~h})$.

${ }^{1} \mathrm{H} \mathrm{NMR}\left(\mathrm{CDCl}_{3}, 500 \mathrm{MHz}\right): \delta=8.01(\mathrm{~d}, J=7.3 \mathrm{~Hz}, 2 \mathrm{H}), 7.73(\mathrm{t}, J=7.7 \mathrm{~Hz}, 1 \mathrm{H}), 7.59(\mathrm{~d}, J=$ $7.8 \mathrm{~Hz}, 1 \mathrm{H}), 7.47(\mathrm{t}, J=7.5 \mathrm{~Hz}, 2 \mathrm{H}), 7.40(\mathrm{t}, J=6.8 \mathrm{~Hz}, 2 \mathrm{H}), 5.12(\mathrm{t}, J=6.8 \mathrm{~Hz}, 1 \mathrm{H}), 4.14(\mathrm{q}, J$ $=6.9 \mathrm{~Hz}, 1 \mathrm{H}), 4.01(\mathrm{q}, J=7.2 \mathrm{~Hz}, 1 \mathrm{H}), 2.47(\mathrm{~m}, 1 \mathrm{H}), 2.15(\mathrm{~m}, 1 \mathrm{H}), 2.00(\mathrm{~m}, 2 \mathrm{H})$.

${ }^{13} \mathrm{C} \mathrm{NMR}\left(126 \mathrm{MHz}, \mathrm{CDCl}_{3}\right): \delta=163.1,156.7,139.7,137.3,128.9,128.8,127.1,118.9,118.3$, $81.7,69.2,33.1,25.9$.

IR: $v=3062,2976,2871,1590,1571,1447,1331,1156,1061,1026,922,815,762,694,623$ $\mathrm{cm}^{-1}$.

HRMS: (ESI) m/z calc. for $\mathrm{C}_{15} \mathrm{H}_{16} \mathrm{NO}(\mathrm{M}+\mathrm{H})$ 226.1232, found 226.1223. 
Spectral Data 
${ }^{1} \mathrm{H}$ NMR Spectrum of 4-(Tetrahydrofuran-2-yl)benzonitrile (2)

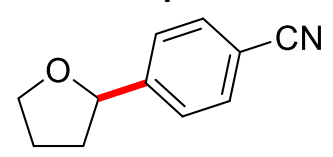

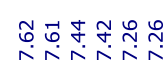

यरV

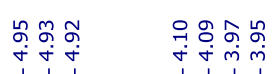

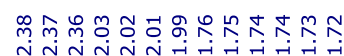
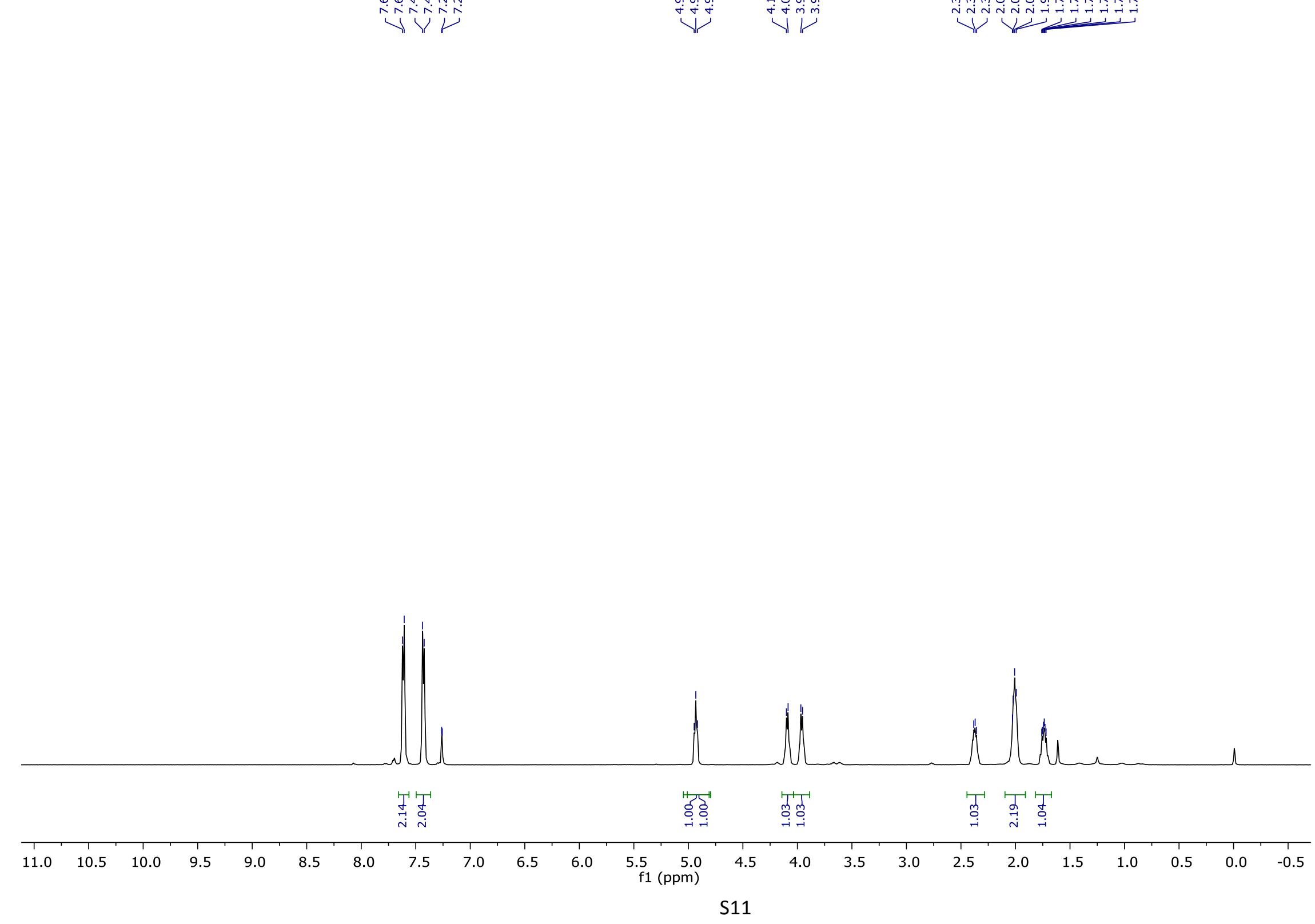
${ }^{13} \mathrm{C}$ NMR Spectrum of 4-(Tetrahydrofuran-2-yl)benzonitrile (2)

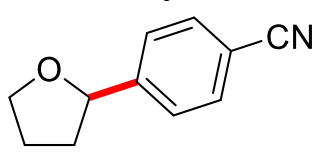

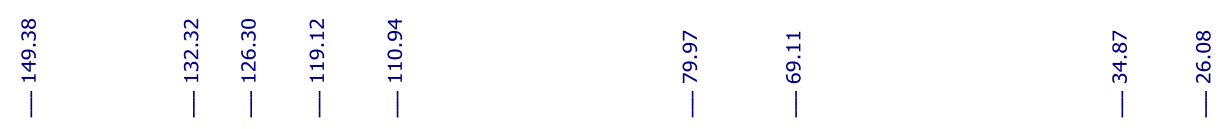

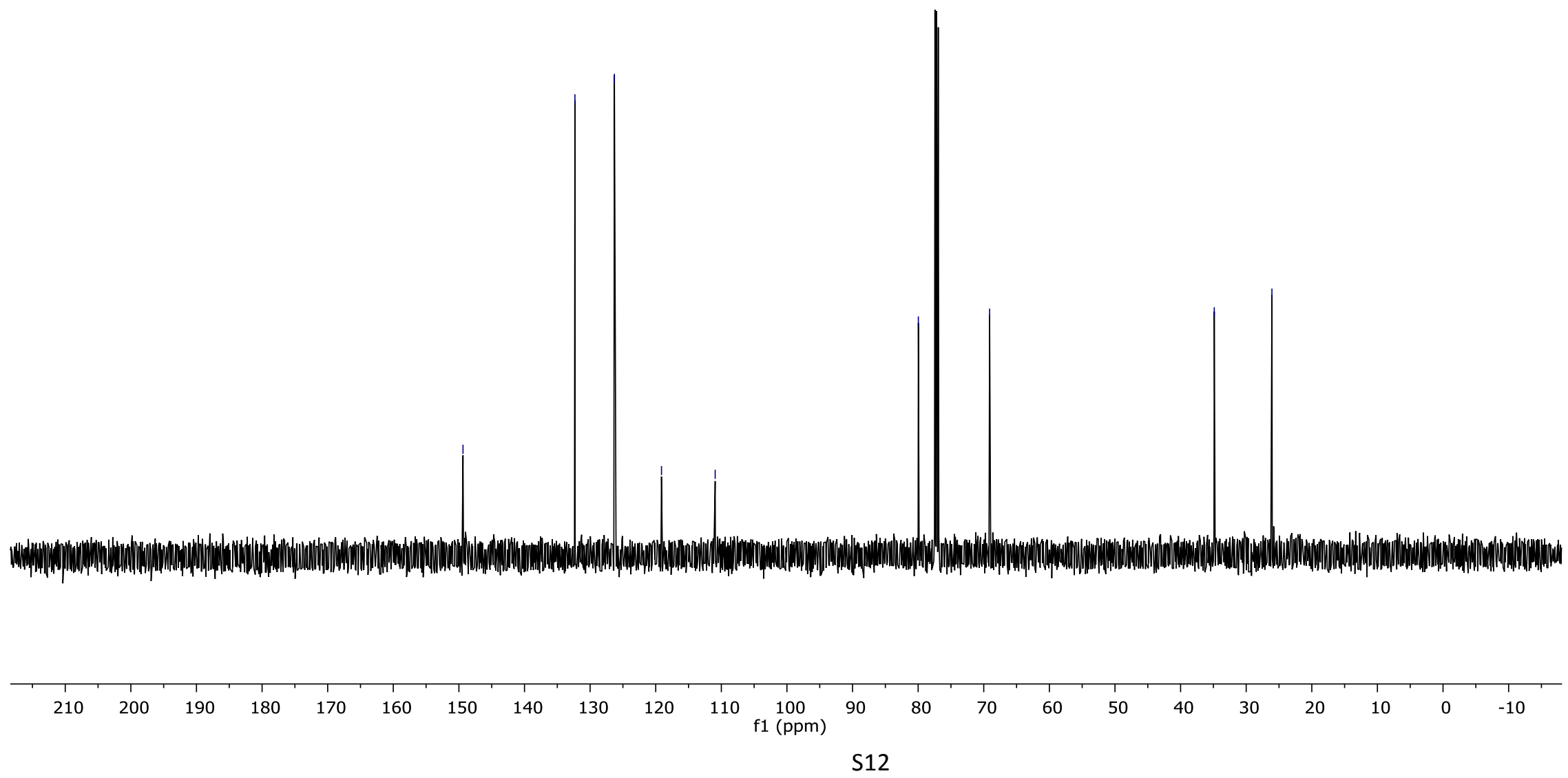


${ }^{1} \mathrm{H}$ NMR Spectrum of 4-(1,4-Dioxan-2-yl)benzonitrile (5)

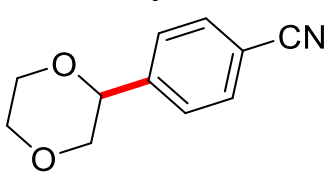

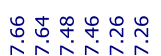

บ๖

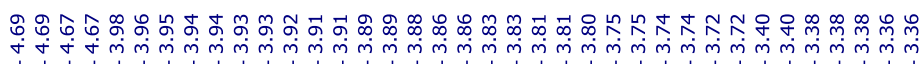

(1)

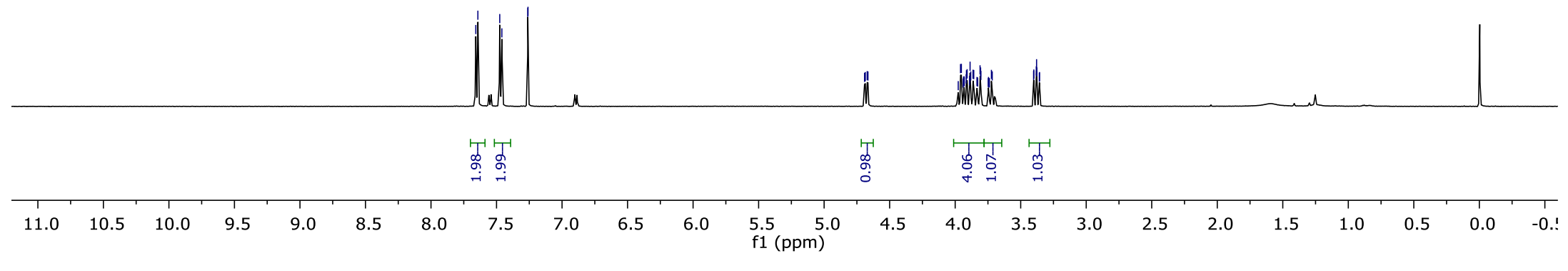


${ }^{13} \mathrm{C}$ NMR Spectrum of 4-(1,4-Dioxan-2-yl)benzonitrile (5)

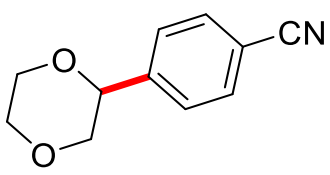

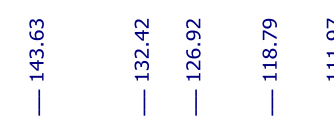
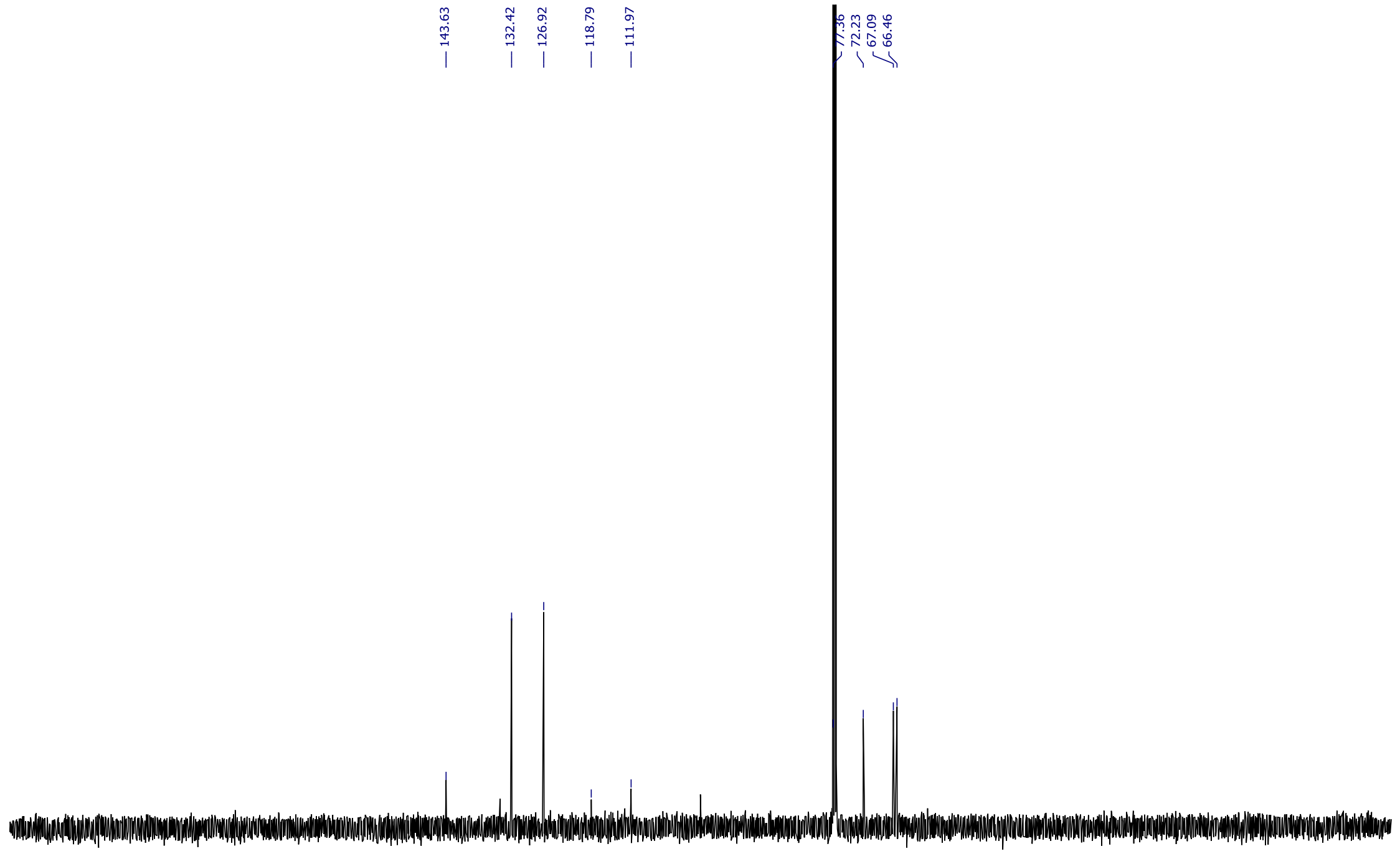

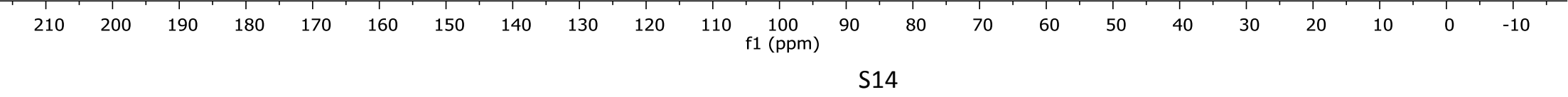


${ }^{1} \mathrm{H}$ NMR Spectrum of 4-(1,2-Dimethoxyethyl)benzonitrile (6)
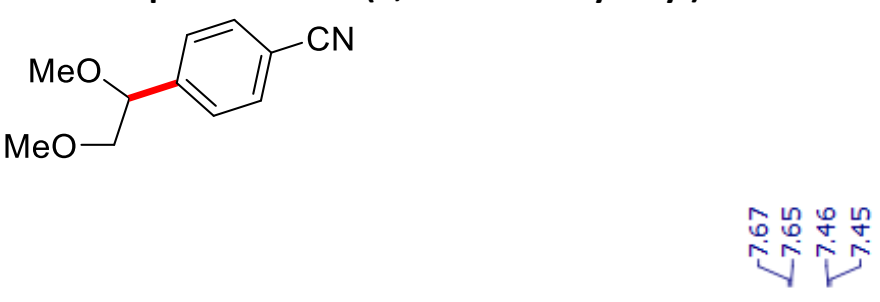

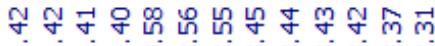

نंj于

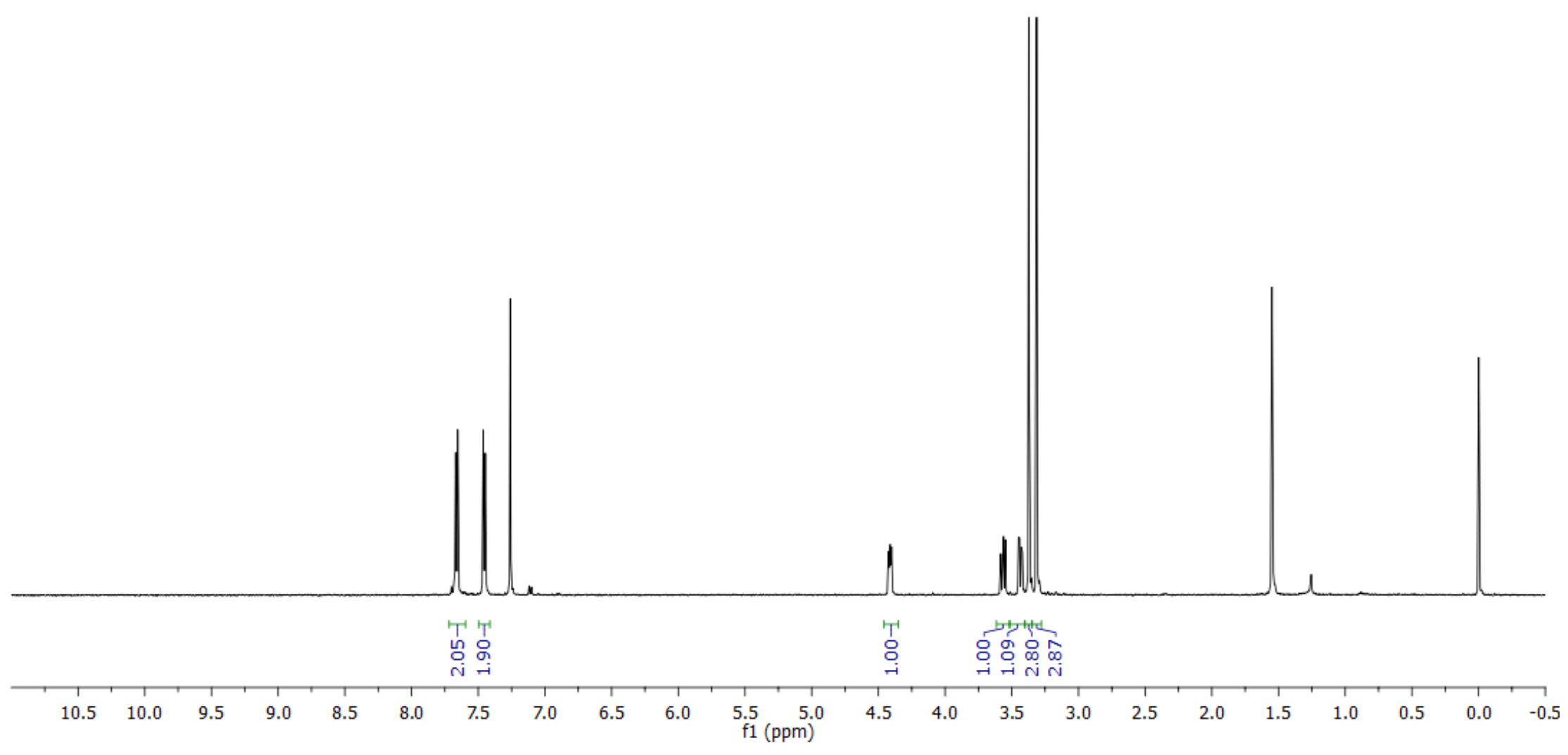


${ }^{13} \mathrm{C}$ NMR Spectrum of 4-(1,2-Dimethoxyethyl)benzonitrile (6)

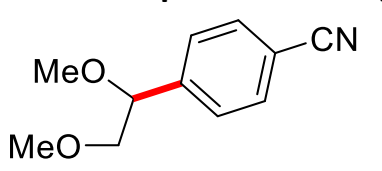

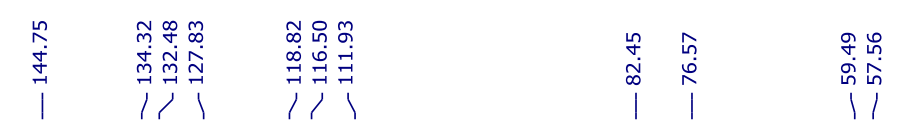

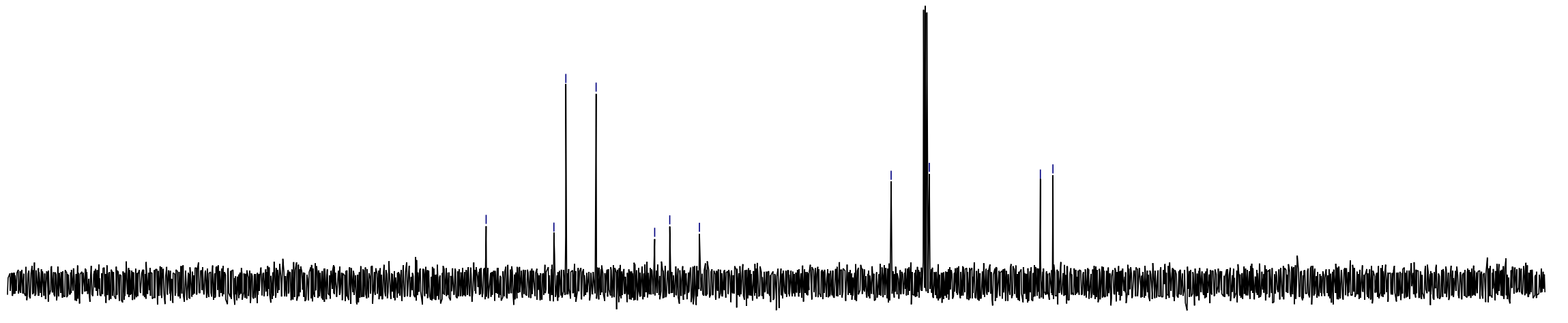

\begin{tabular}{|c|c|c|c|c|c|c|c|c|c|c|c|c|c|c|c|c|c|c|c|c|c|c|}
\hline 210 & 200 & 190 & 180 & 170 & 160 & 150 & 140 & 130 & 120 & 110 & $\begin{array}{c}100 \\
\mathrm{f} 1(\mathrm{ppm})\end{array}$ & $\begin{array}{l}90 \\
\text { S16 }\end{array}$ & 80 & 70 & 60 & 50 & 40 & 30 & 20 & 10 & 0 & -10 \\
\hline
\end{tabular}


${ }^{1} \mathrm{H}$ NMR Spectrum of 4-(1-Ethoxyethyl)benzonitrile (7)

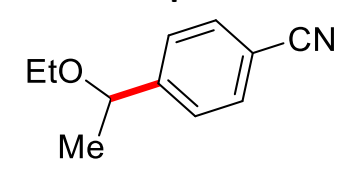

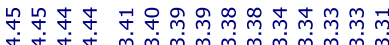

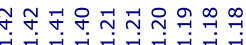

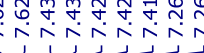

4
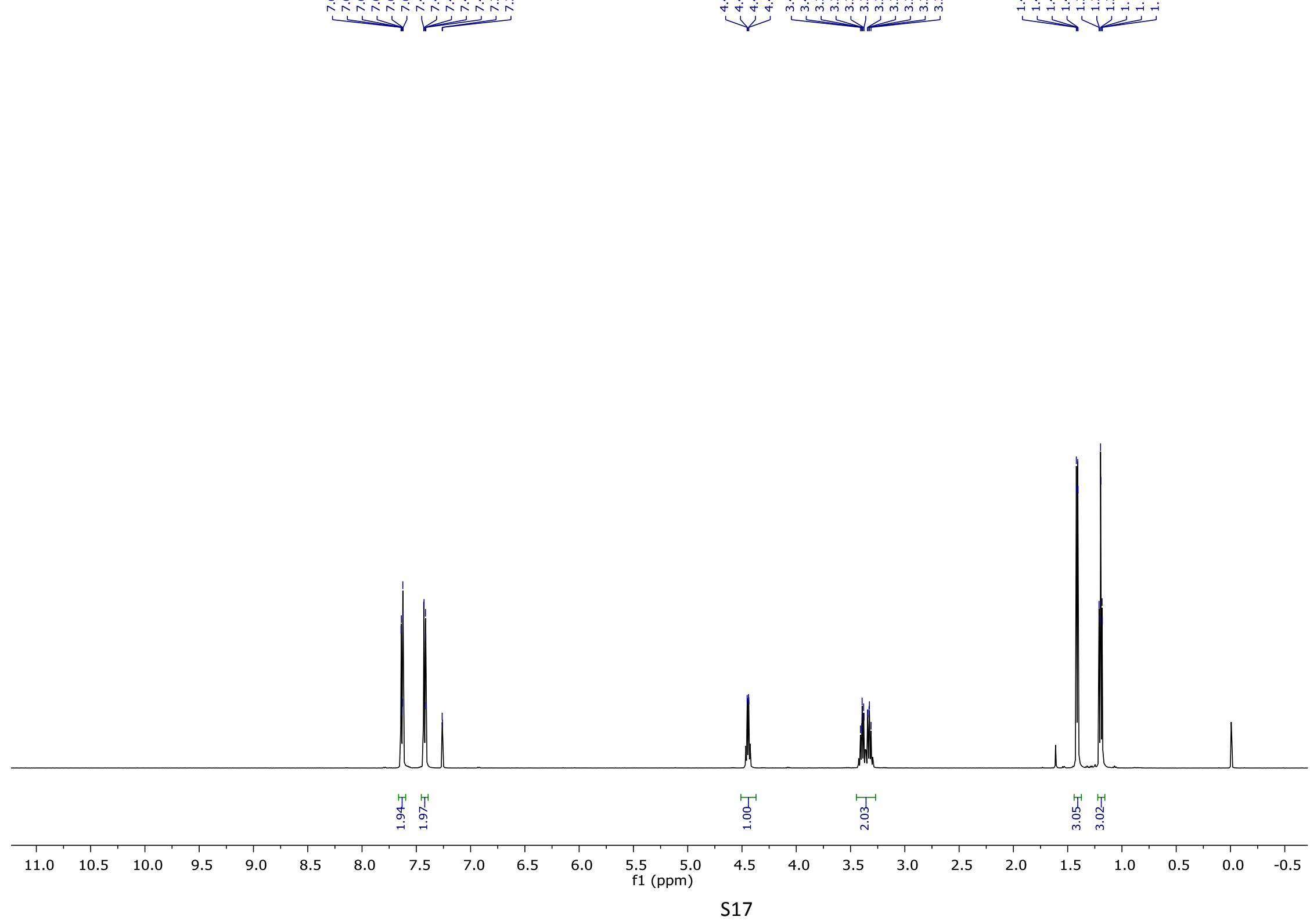
${ }^{13}$ C NMR Spectrum of 4-(1-Ethoxyethyl)benzonitrile (7)

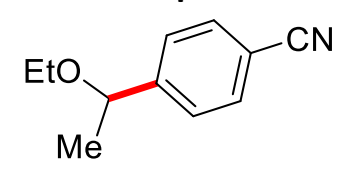

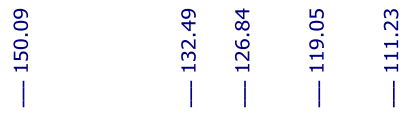

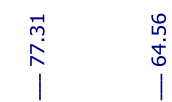

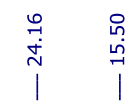

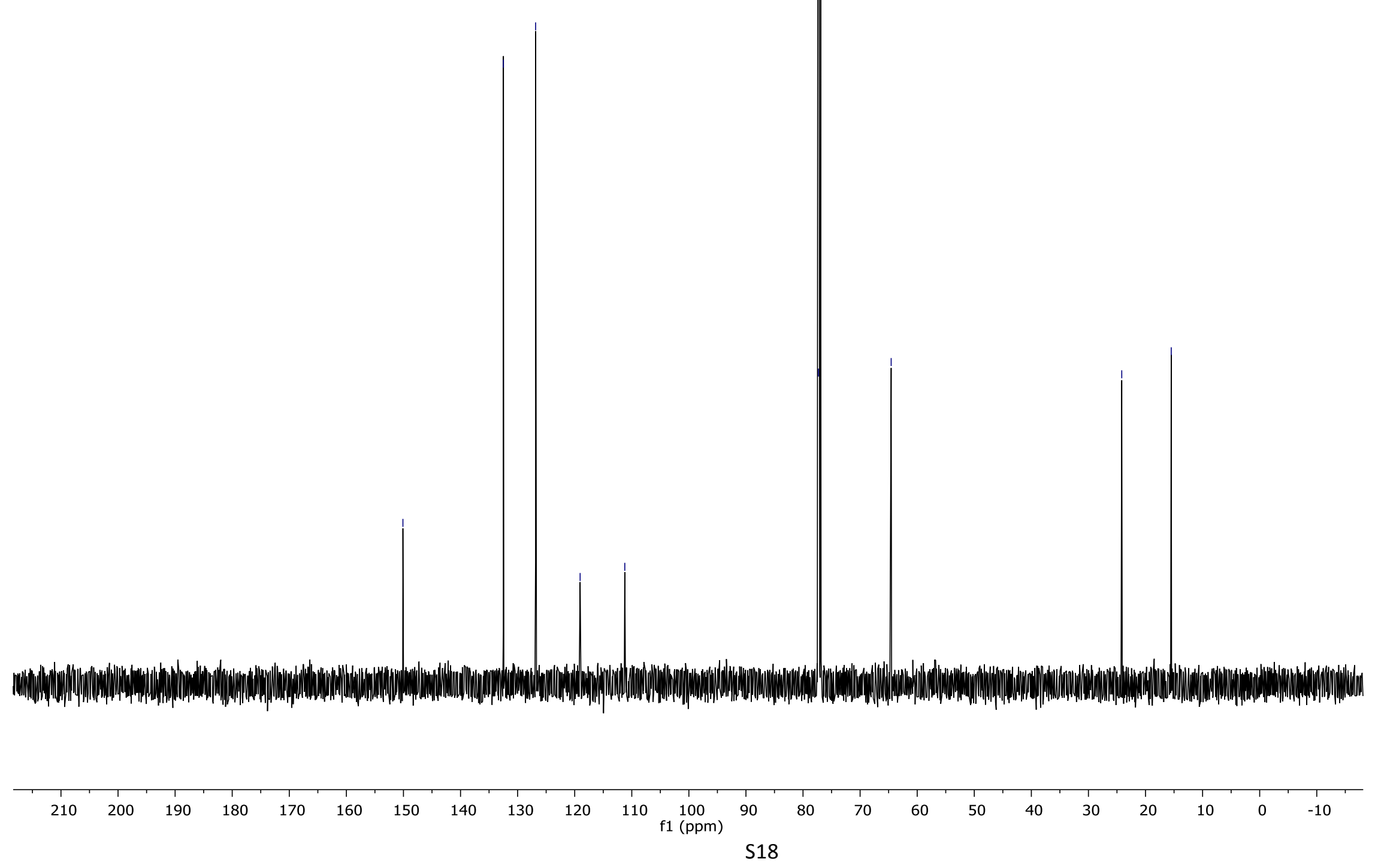


${ }^{1}$ H NMR Spectrum of 4-(Tetrahydrothiophen-2-yl)benzonitrile (9)

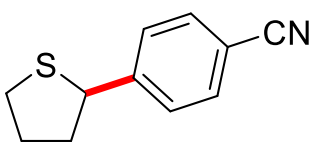

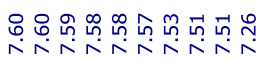

ininining

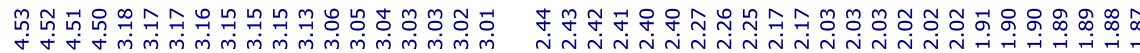

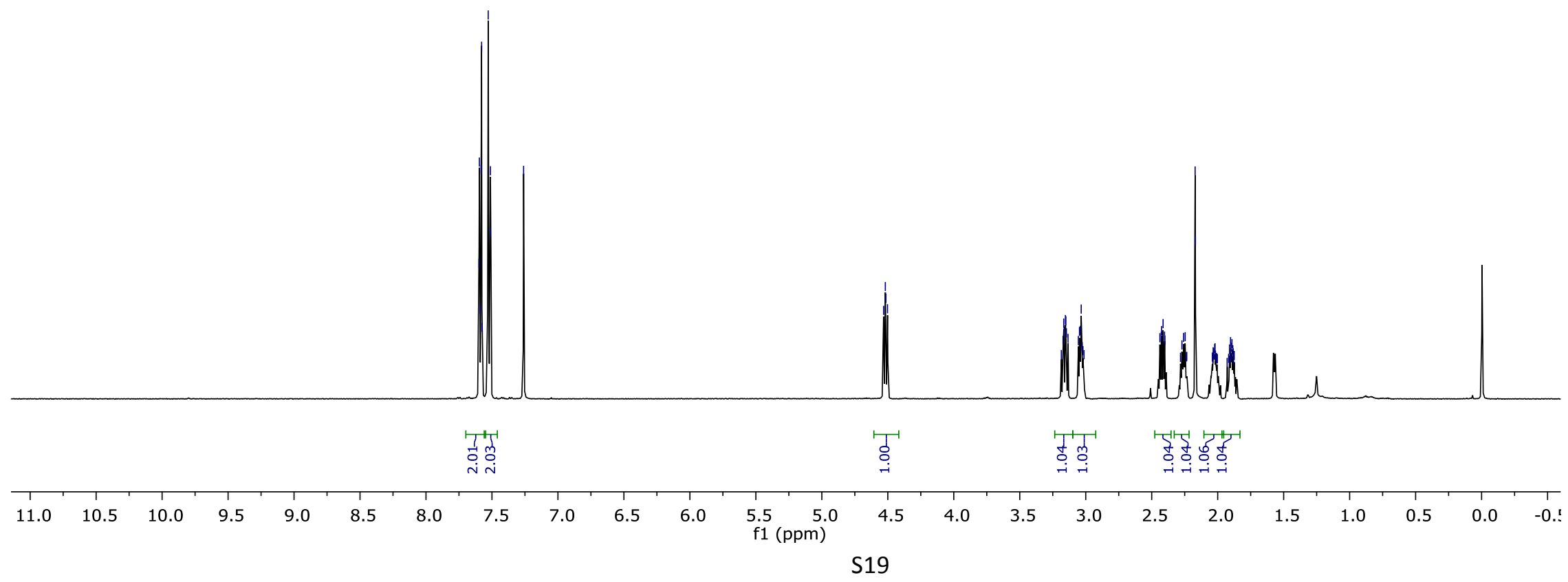


${ }^{13} \mathrm{C}$ NMR Spectrum of 4-(Tetrahydrothiophen-2-yl)benzonitrile (9)

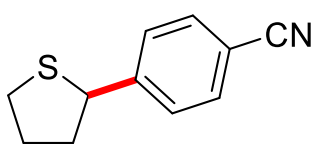

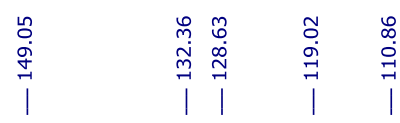

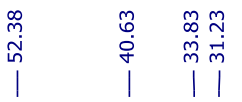

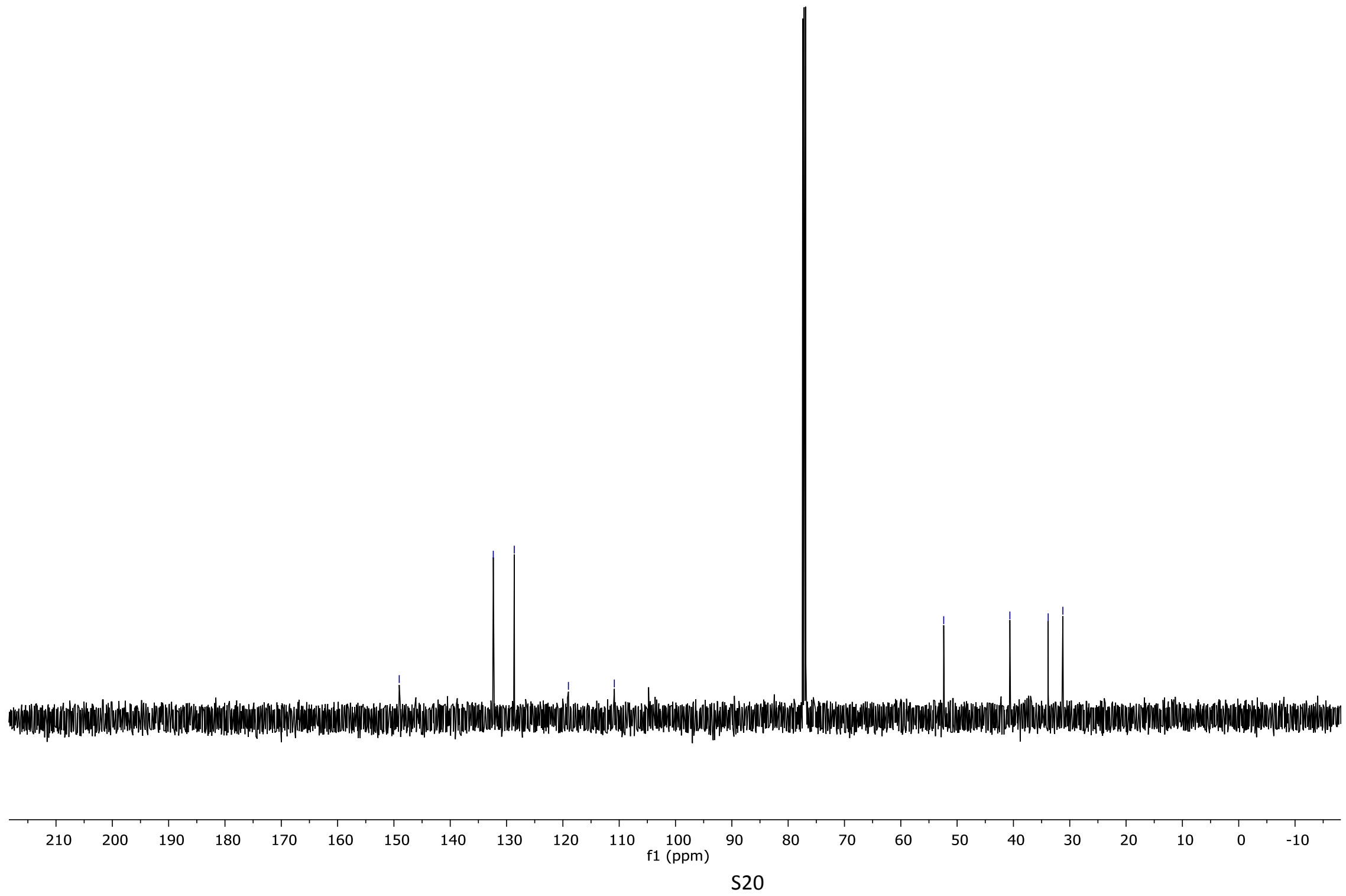


${ }^{1} \mathrm{H}$ NMR Spectrum of 4-(1-Methyl-5-oxopyrrolidin-2-yl)benzonitrile (10)
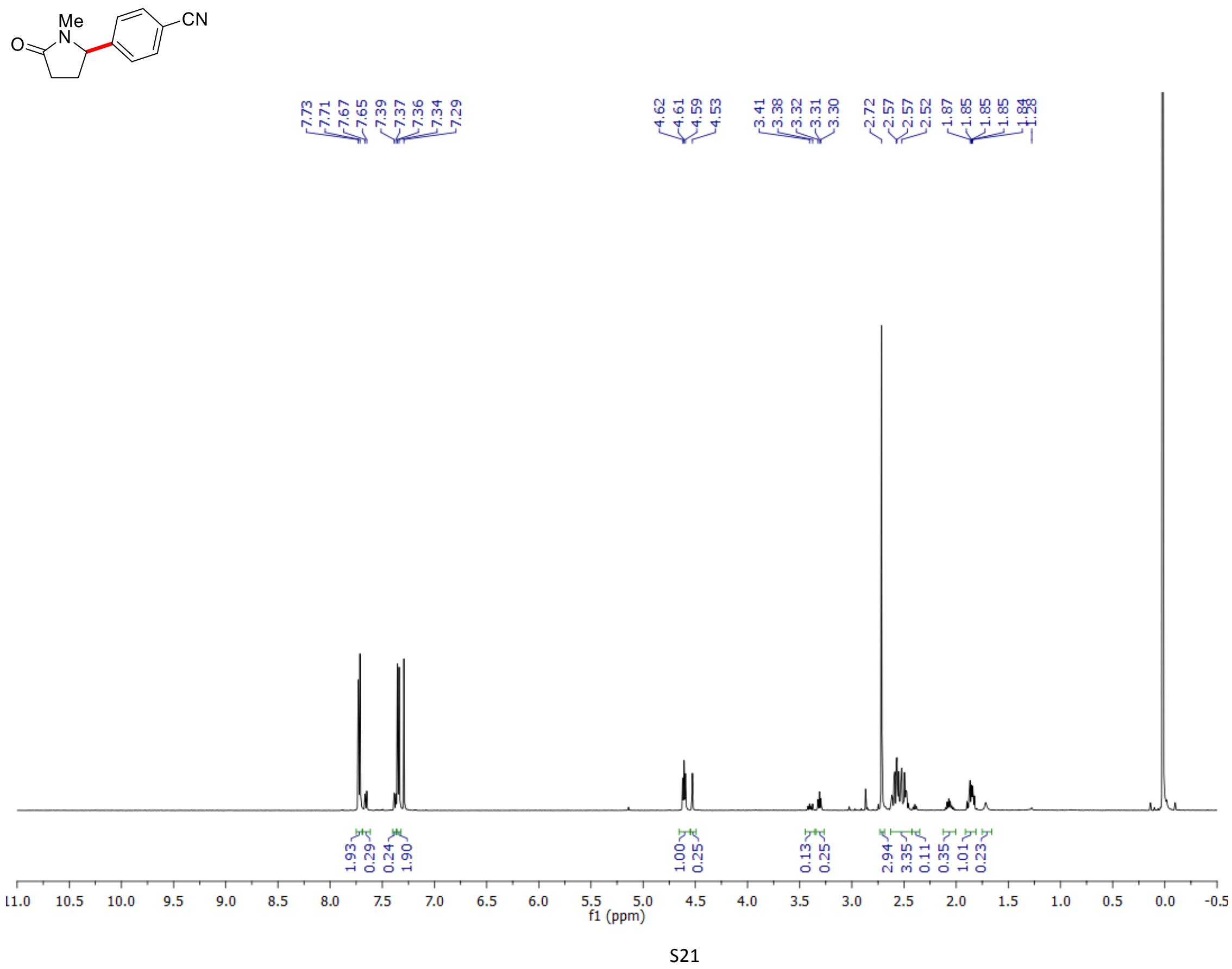
${ }^{13}$ C NMR Spectrum of 4-(1-Methyl-5-oxopyrrolidin-2-yl)benzonitrile (10)

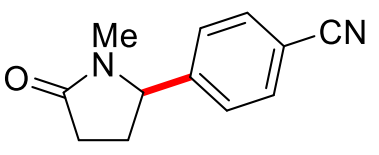

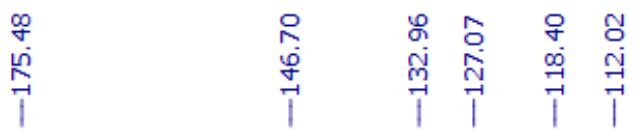

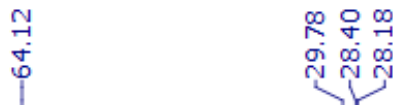

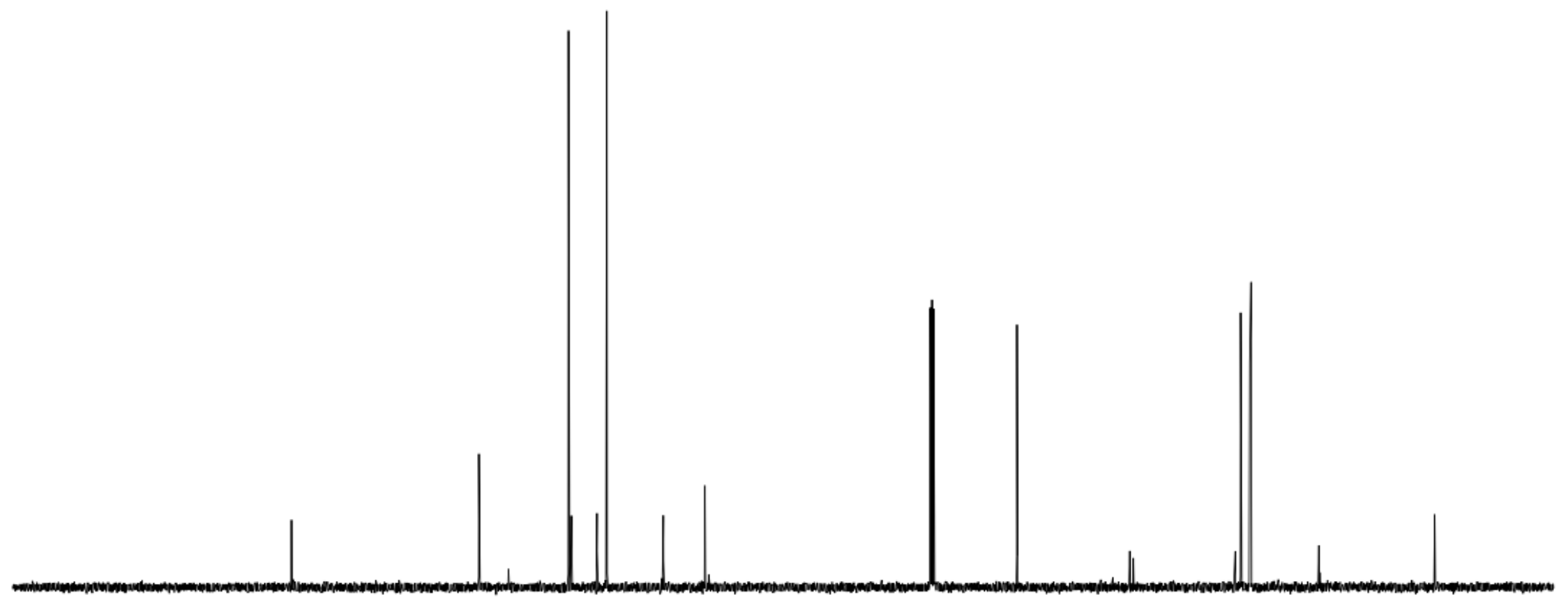

$210 \quad 200$

$190 \quad 180 \quad 170$

160

$150 \quad 140$

$130 \quad 120$

$110 \stackrel{100}{100}$

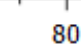

8070

60 
${ }^{1} \mathrm{H}$ NMR Spectrum of 4-Benzylbenzonitrile (11)

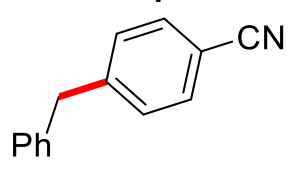

象

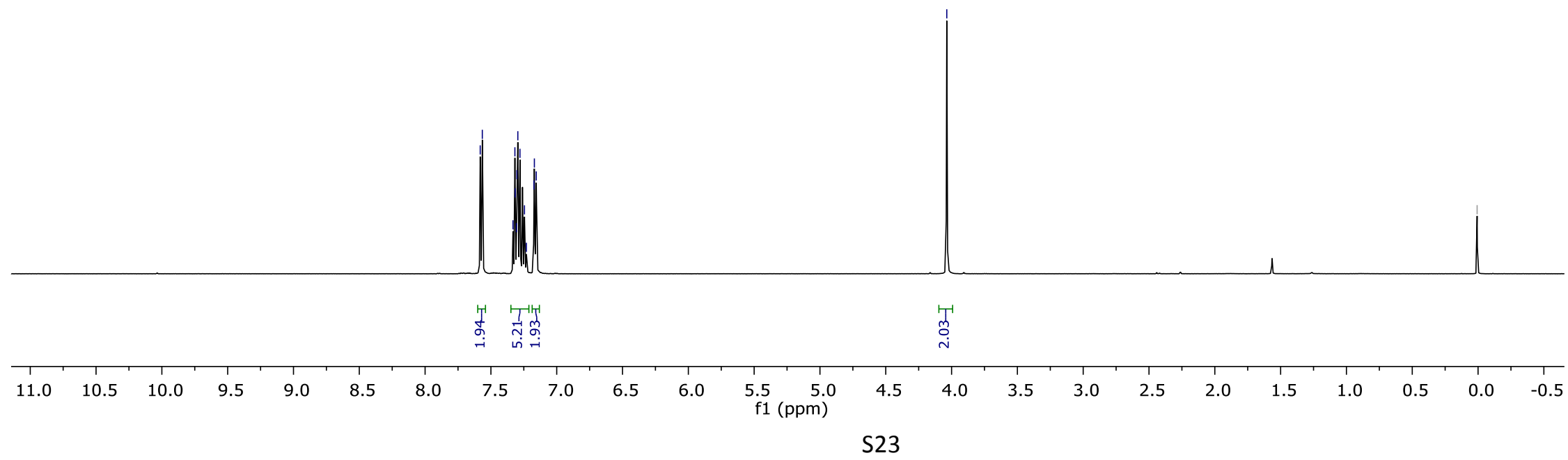


${ }^{13} \mathrm{C}$ NMR Spectrum of 4-Benzylbenzonitrile (11)

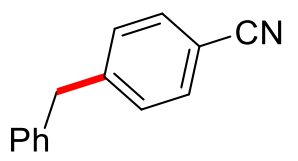

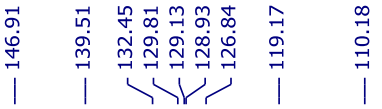

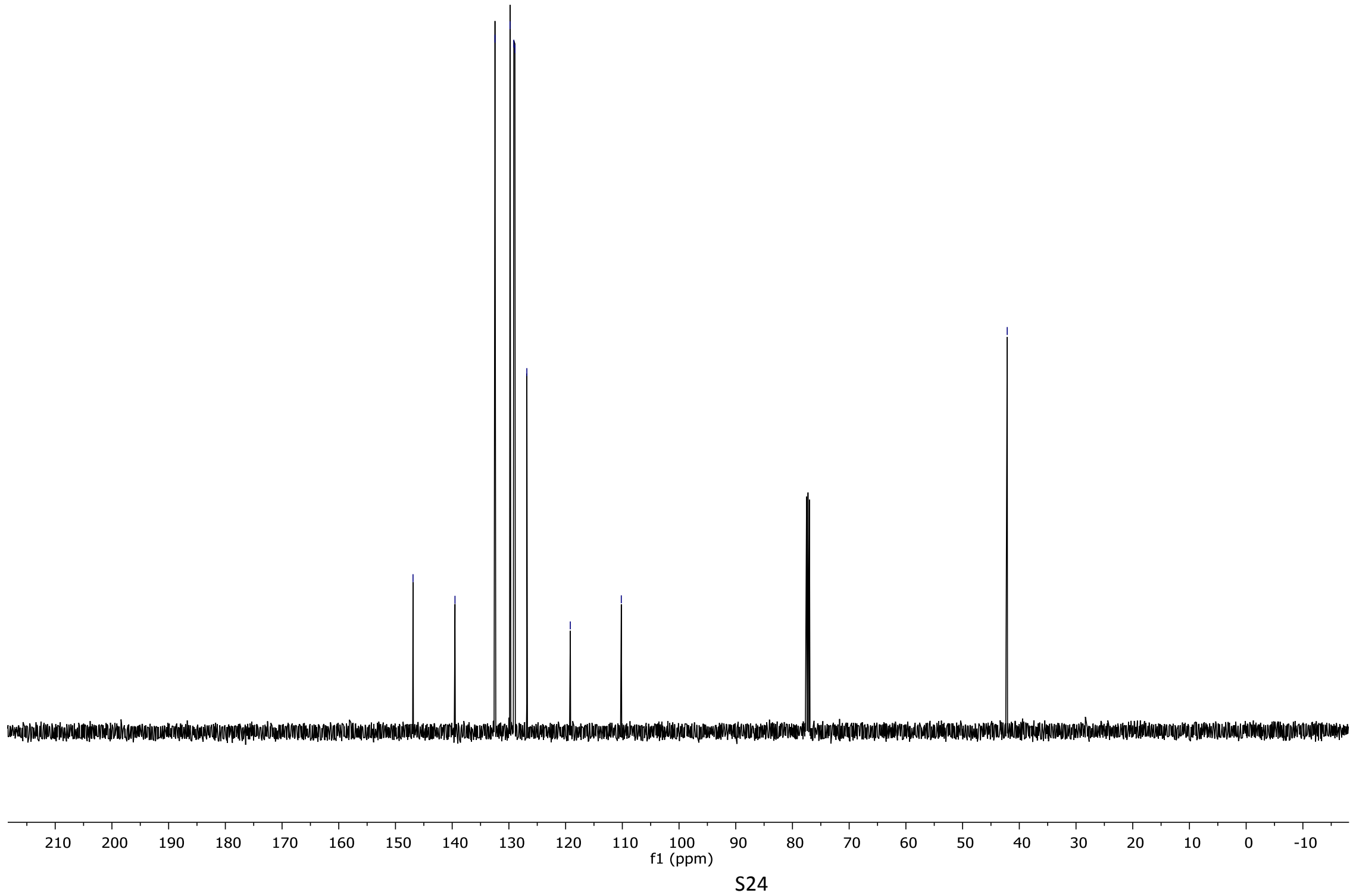


${ }^{1} \mathrm{H}$ NMR Spectrum of Methyl 4-(Tetrahydrofuran-2-yl)benzoate (13)
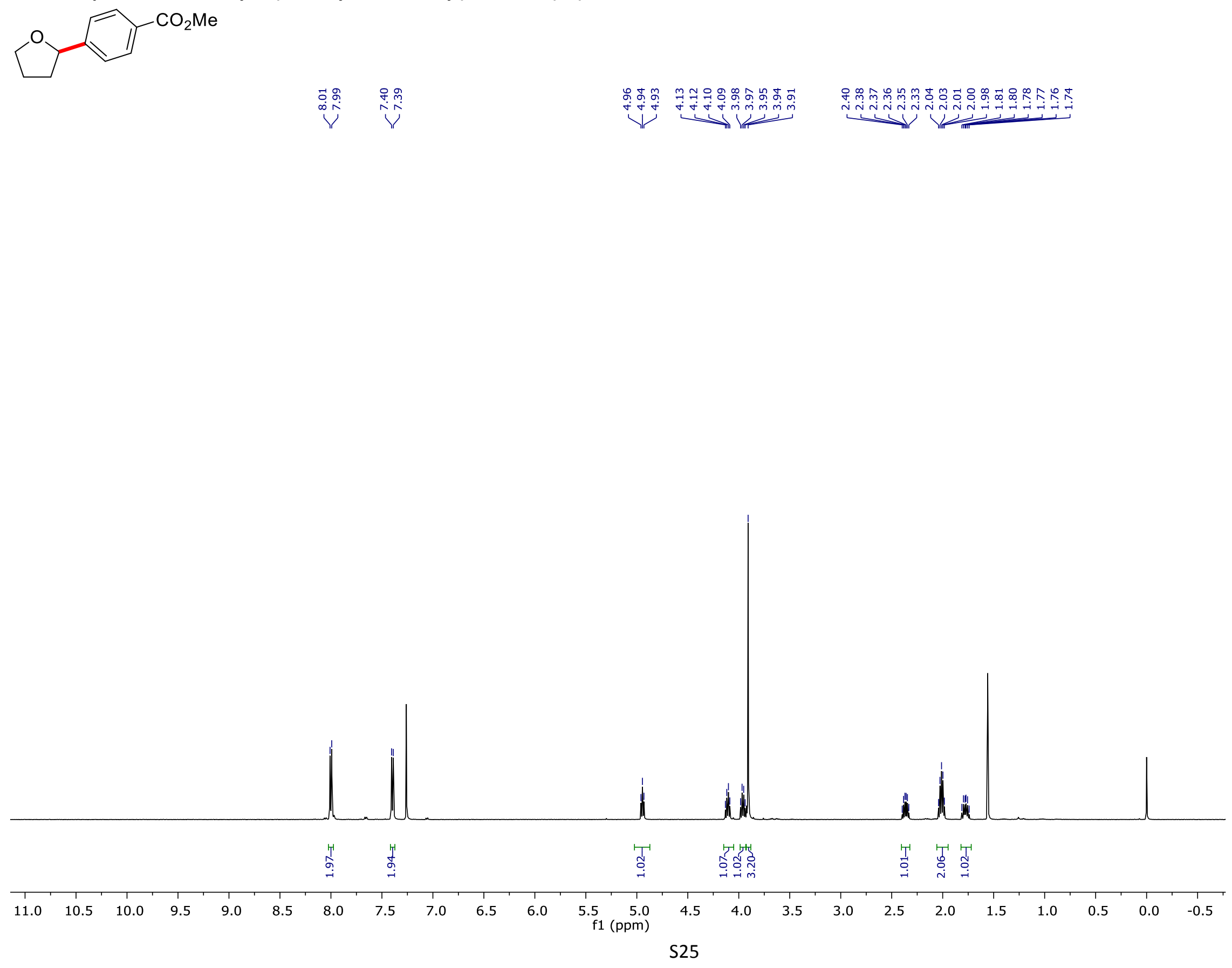
${ }^{13} \mathrm{C}$ NMR Spectrum of Methyl 4-(Tetrahydrofuran-2-yl)benzoate (13)

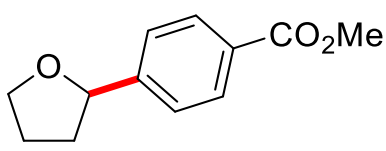

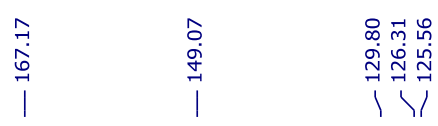

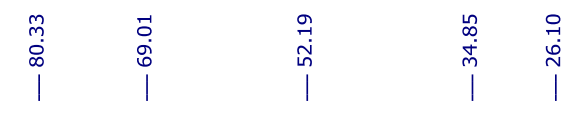

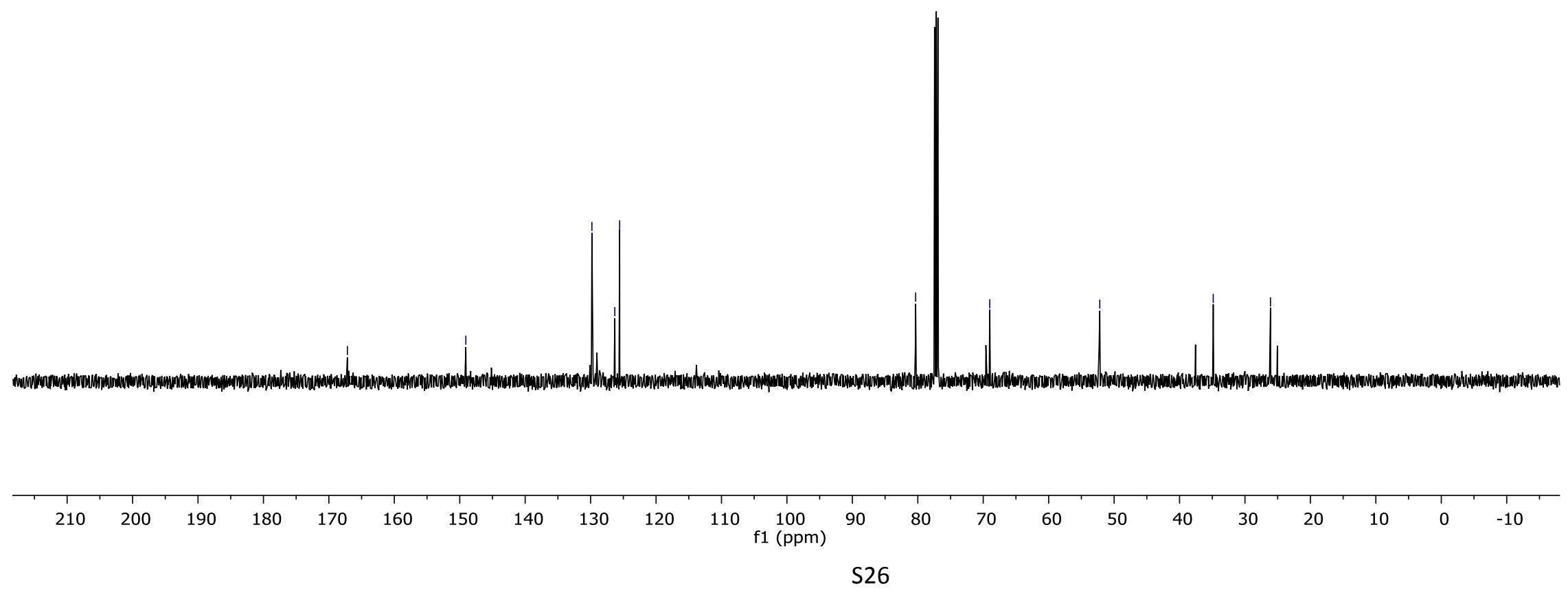


${ }^{1} \mathrm{H}$ NMR Spectrum of 1-(4-(Tetrahydrofuran-2-yl)phenyl)ethan-1-one (14)
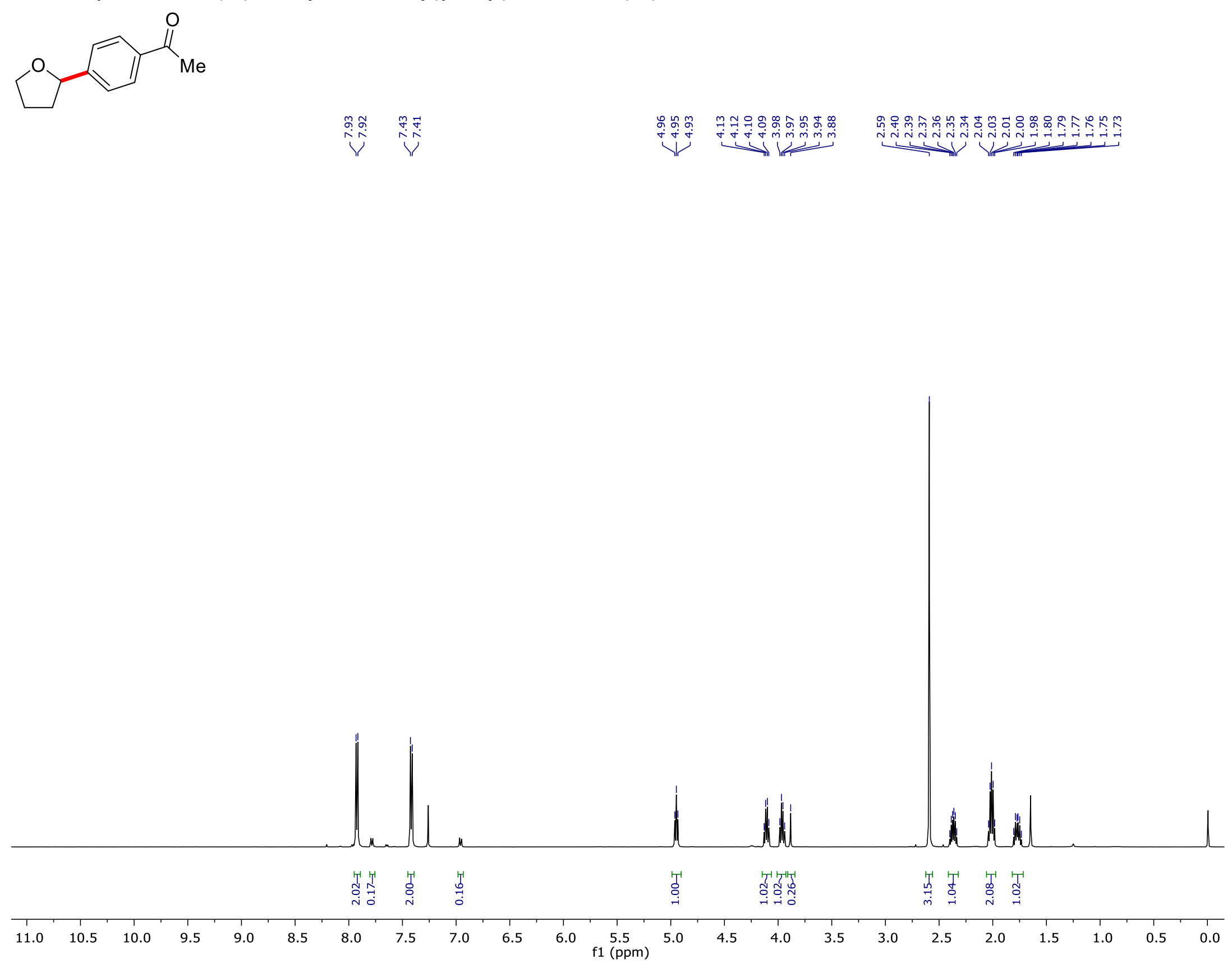
${ }^{13} \mathrm{C}$ NMR Spectrum of 1-(4-(Tetrahydrofuran-2-yl)phenyl)ethan-1-one (14)

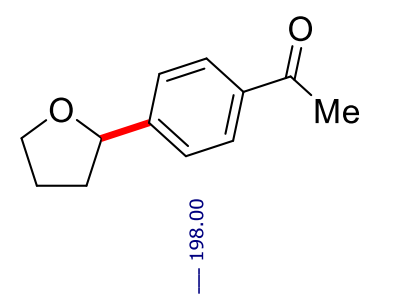

票

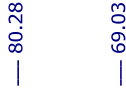

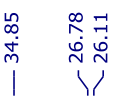

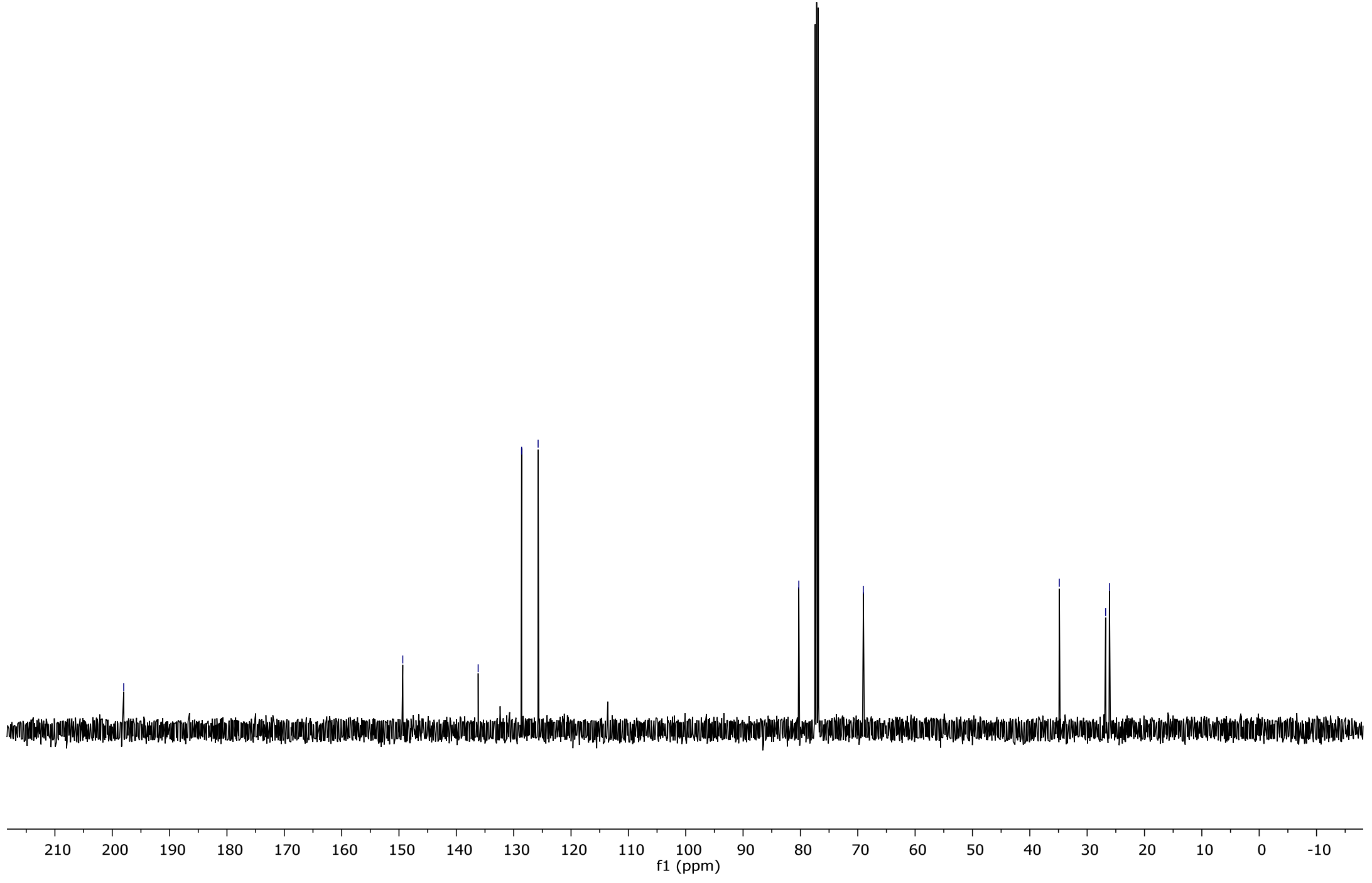


${ }^{1} \mathrm{H}$ NMR Spectrum of 4-(Tetrahydrofuran-2-yl)benzaldehyde (15)
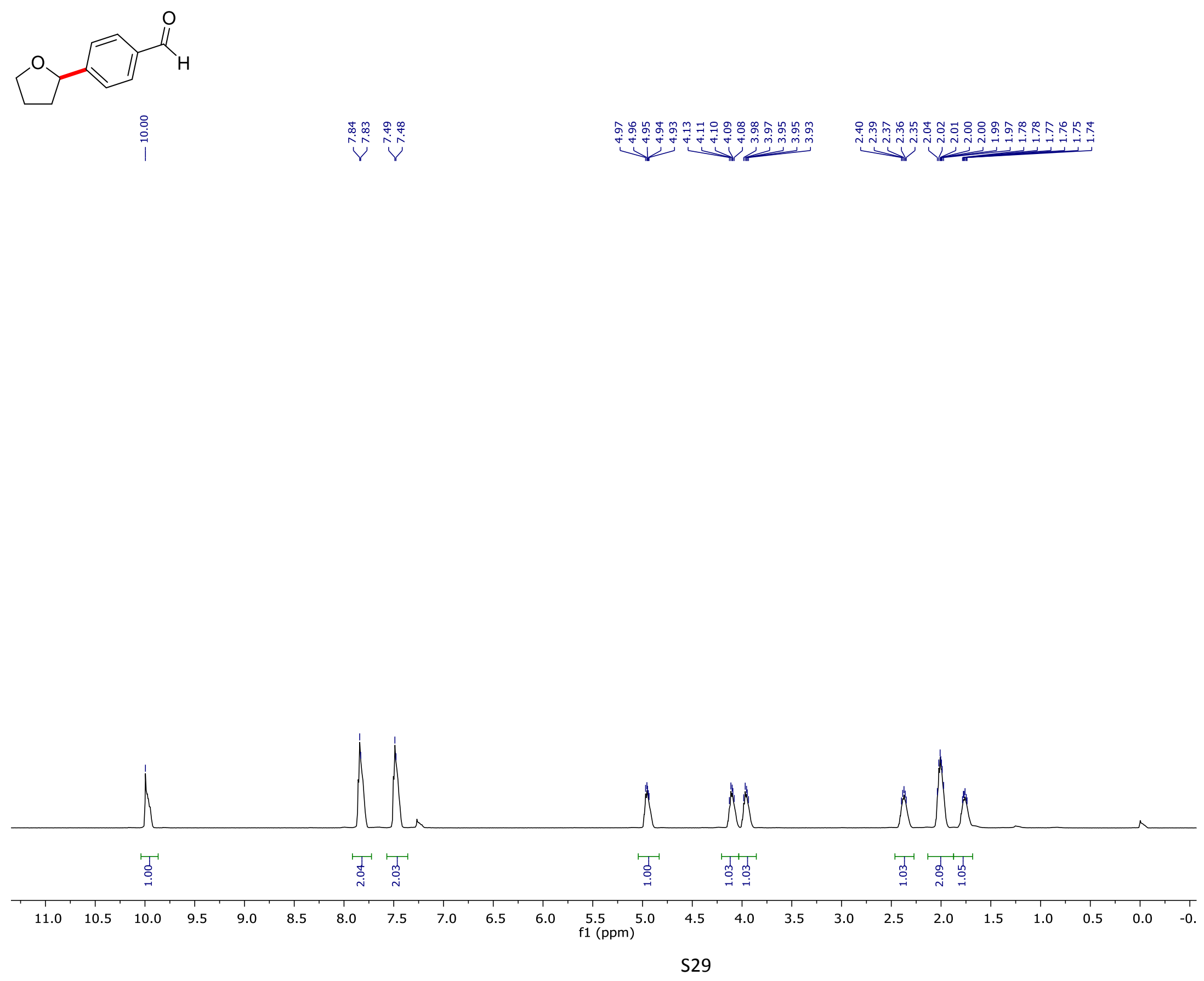
${ }^{13} \mathrm{C}$ NMR Spectrum of 4-(Tetrahydrofuran-2-yl)benzaldehyde (15)

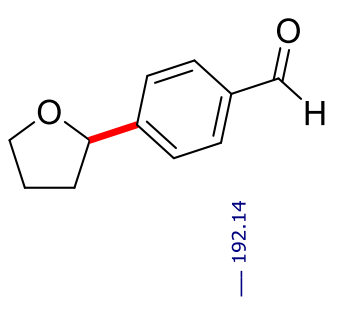

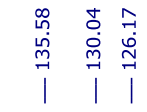

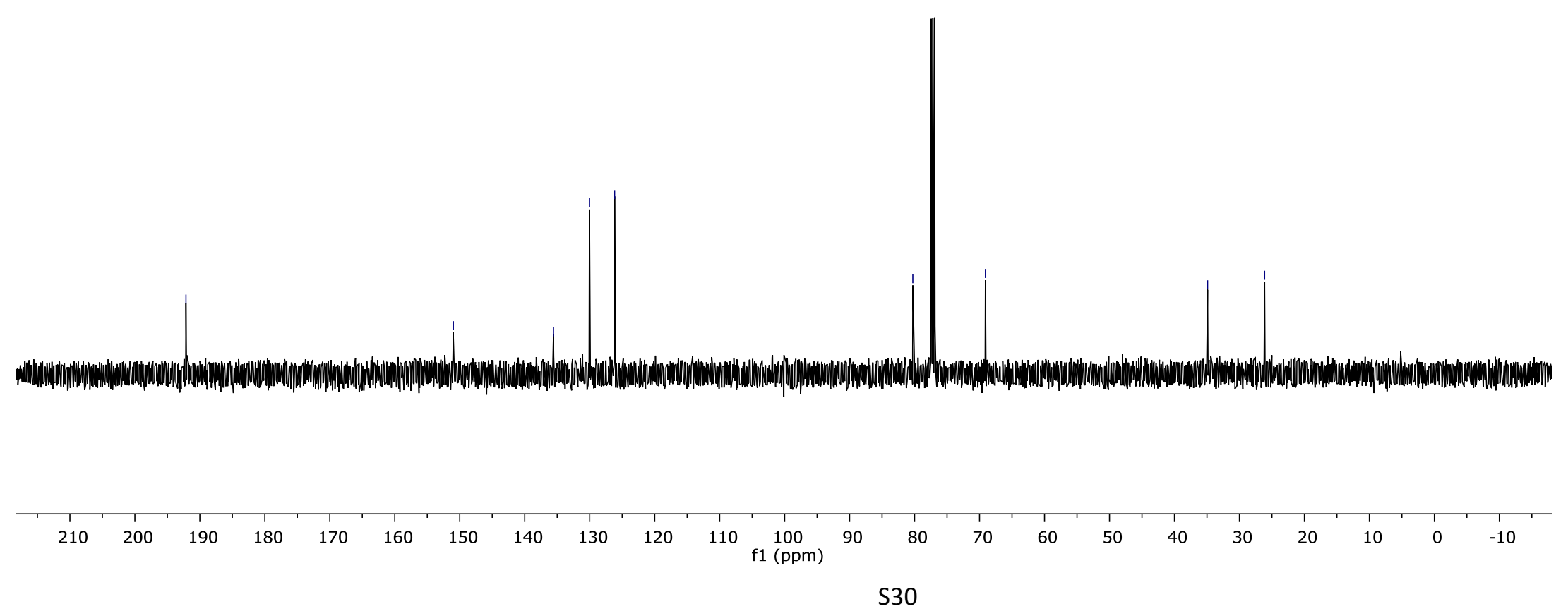


${ }^{1} \mathrm{H}$ NMR Spectrum of 2-(4-(trifluoromethyl)phenyl)tetrahydrofuran (16)

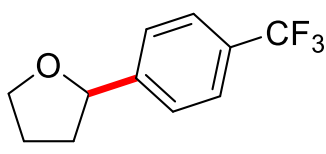

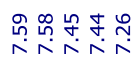

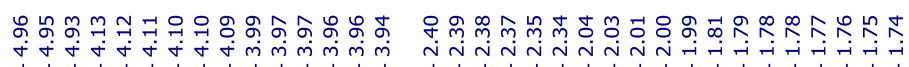

प|

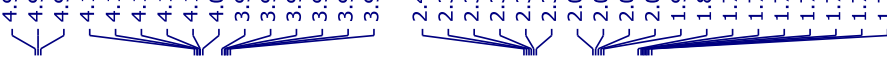

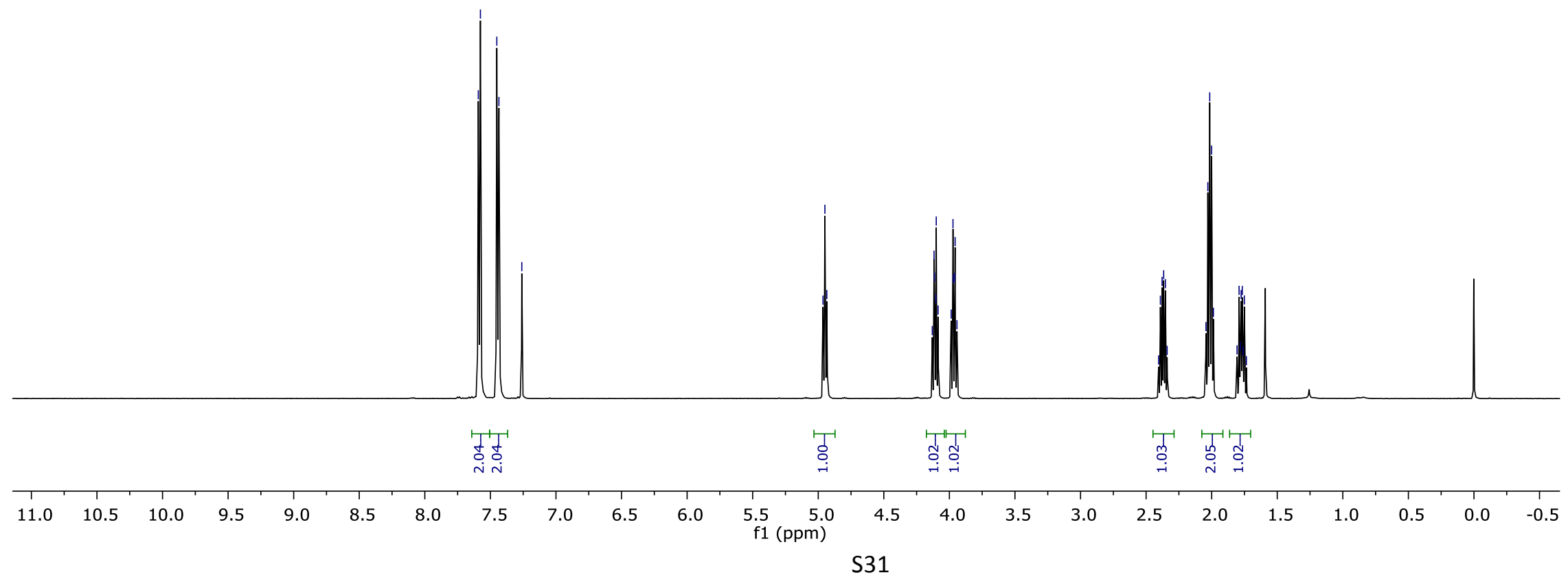


${ }^{13} \mathrm{C}$ NMR Spectrum of 2-(4-(trifluoromethyl)phenyl)tetrahydrofuran (16)
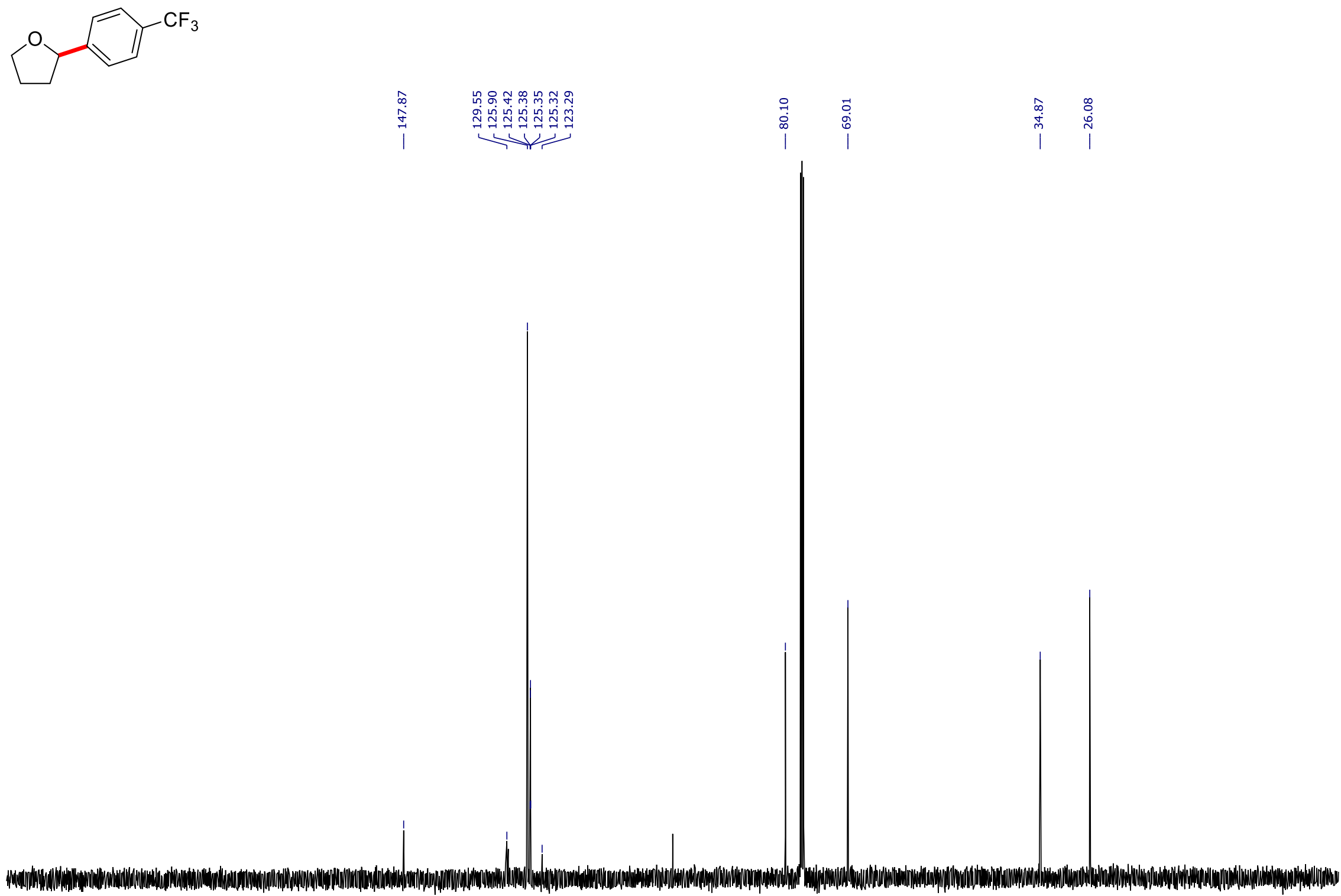
${ }^{19}$ F NMR Spectrum of 2-(4-(trifluoromethyl)phenyl)tetrahydrofuran (16)

COCF

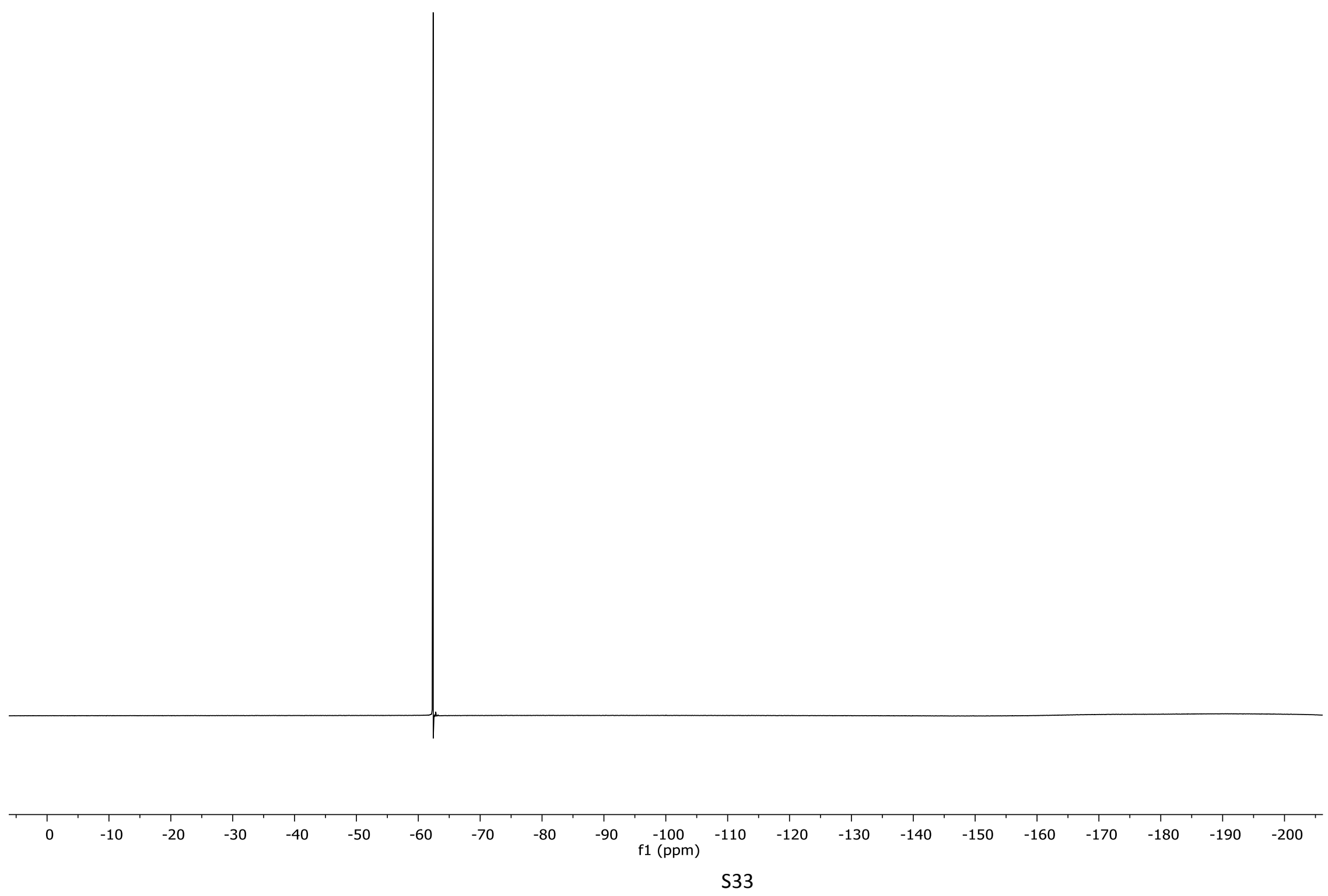


${ }^{1} \mathrm{H}$ NMR Spectrum of 2-(4-Chlorophenyl)tetrahydrofuran (17)
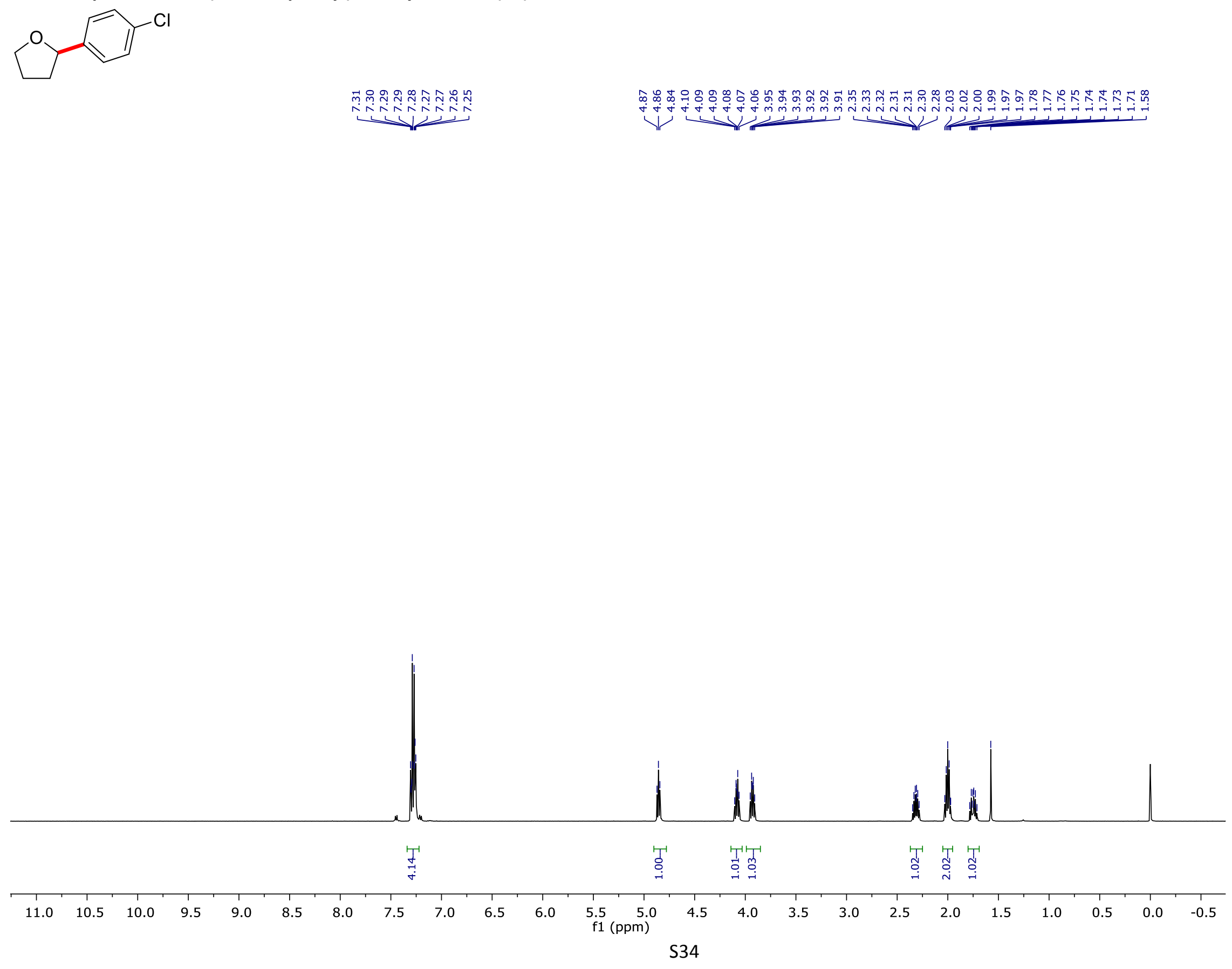
${ }^{13} \mathrm{C}$ NMR Spectrum of 2-(4-Chlorophenyl)tetrahydrofuran (17)
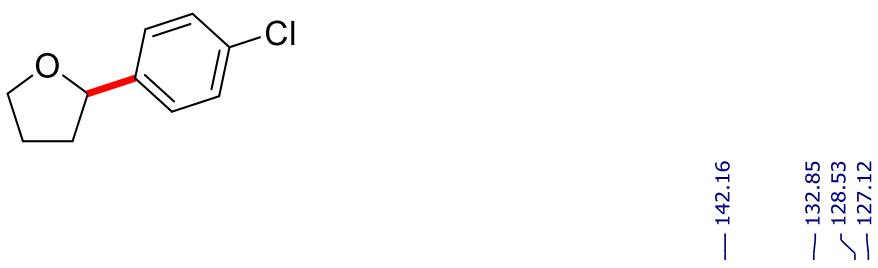

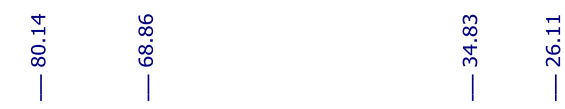

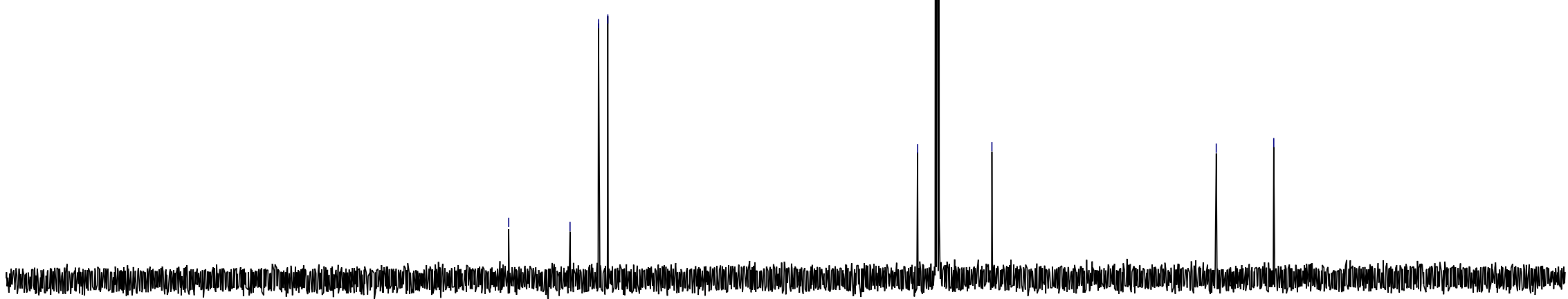

$\begin{array}{llllllllllll}210 & 200 & 190 & 180 & 170 & 160 & 150 & 140 & 130 & 120 & 110 & \begin{array}{l}100 \\ \mathrm{f} 1(\mathrm{ppm})\end{array}\end{array}$


${ }^{1} \mathrm{H}$ NMR Spectrum of 2-(4-Methoxyphenyl)tetrahydrofuran (18)

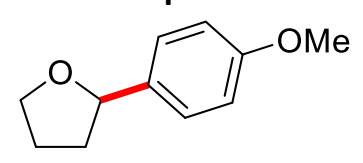

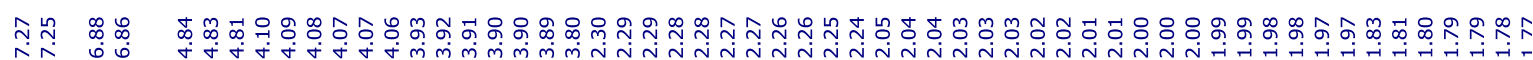

$Y$ Y

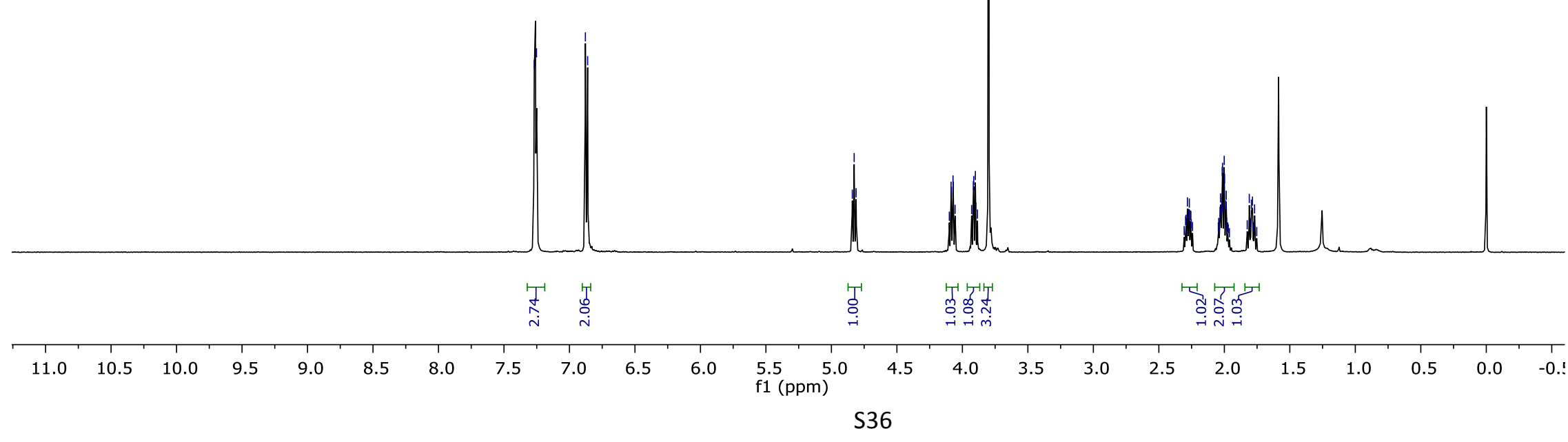


${ }^{13} \mathrm{C}$ NMR Spectrum of 2-(4-Methoxyphenyl)tetrahydrofuran (18)<smiles>COc1ccc(C2CCCO2)cc1</smiles>
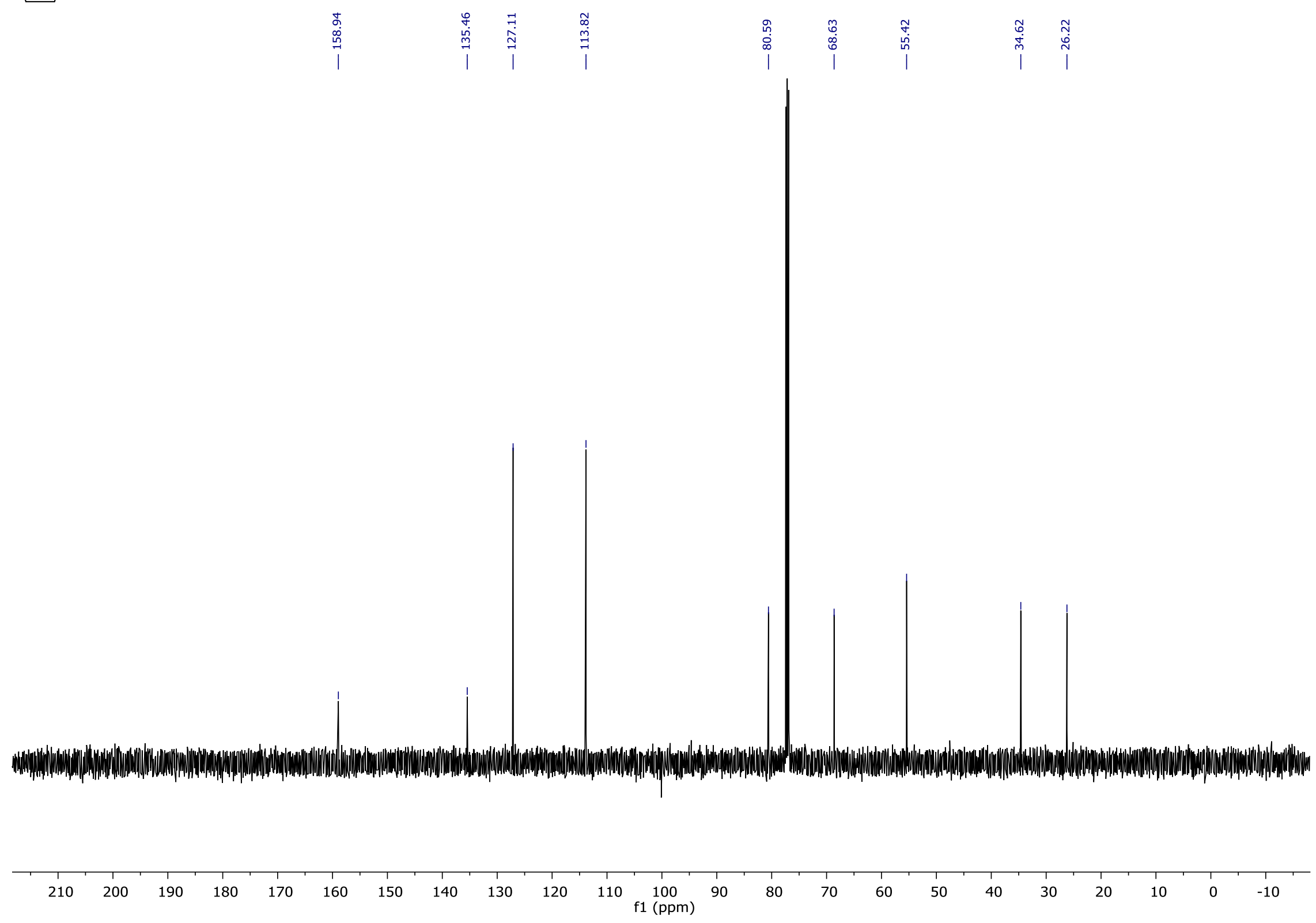

S37 
${ }^{1}$ H NMR Spectrum of 2-(3-Methoxyphenyl)tetrahydrofuran (19)
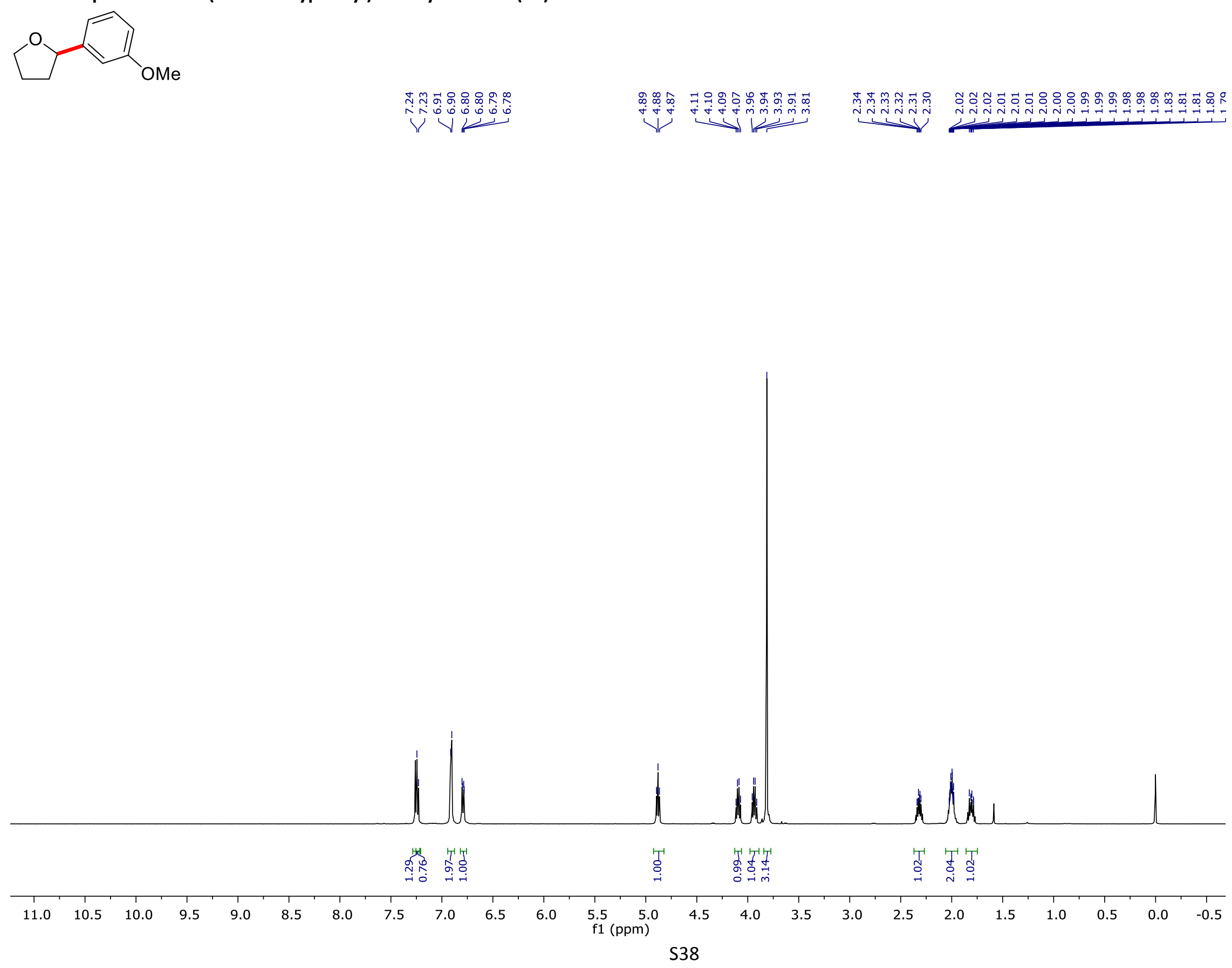
${ }^{13}$ C NMR Spectrum of 2-(3-Methoxyphenyl)tetrahydrofuran (19)

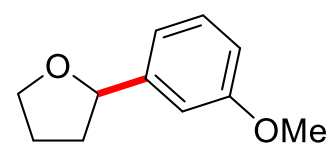

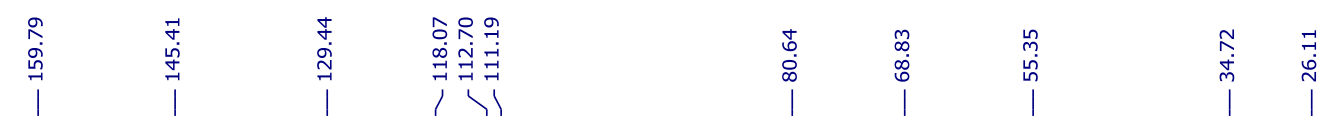

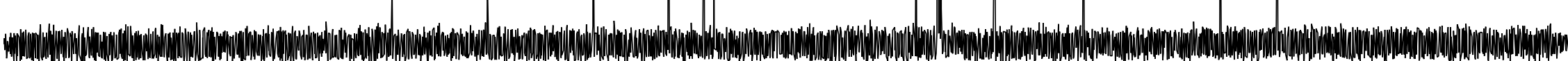

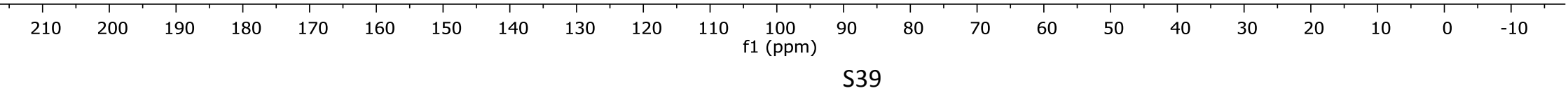


${ }^{1}$ H NMR Spectrum of 5-(Tetrahydrofuran-2-yl)quinoline (20)
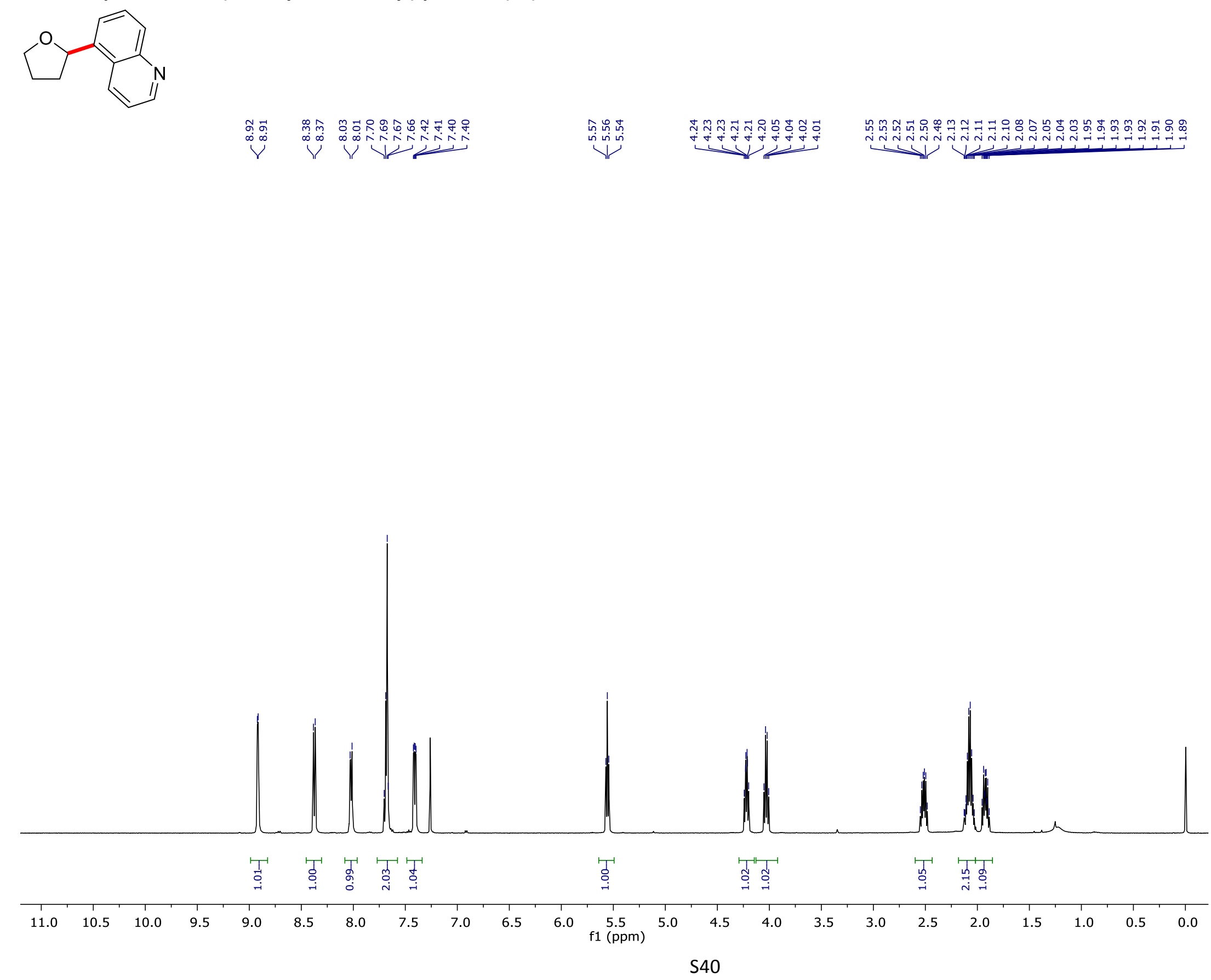
${ }^{13}$ C NMR Spectrum of 5-(Tetrahydrofuran-2-yl)quinoline (20)

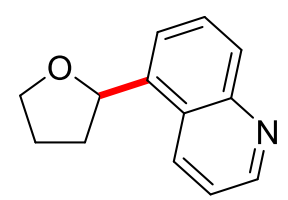

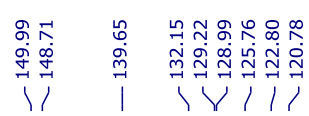

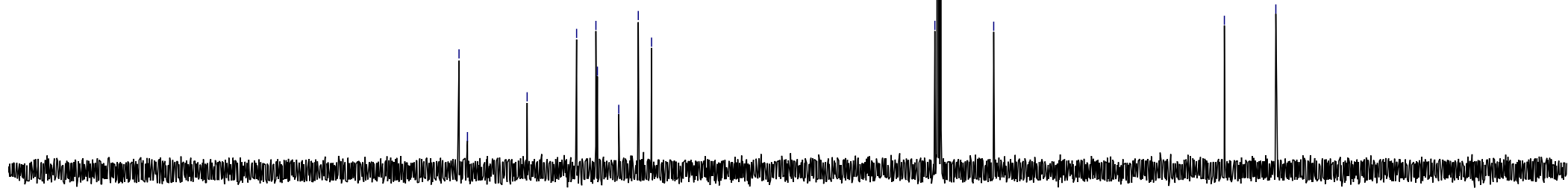


${ }^{1} \mathrm{H}$ NMR Spectrum of 5-(Tetrahydrofuran-2-yl)thiophene-2-carbaldehyde (21)
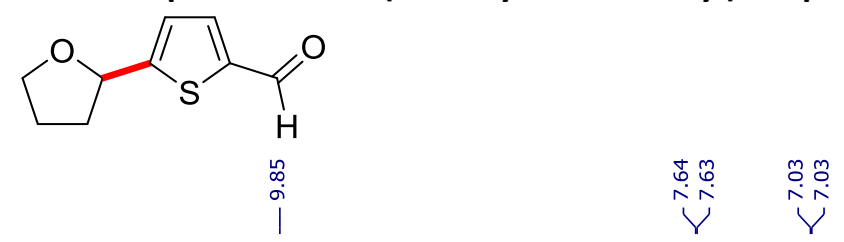

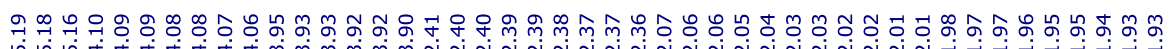

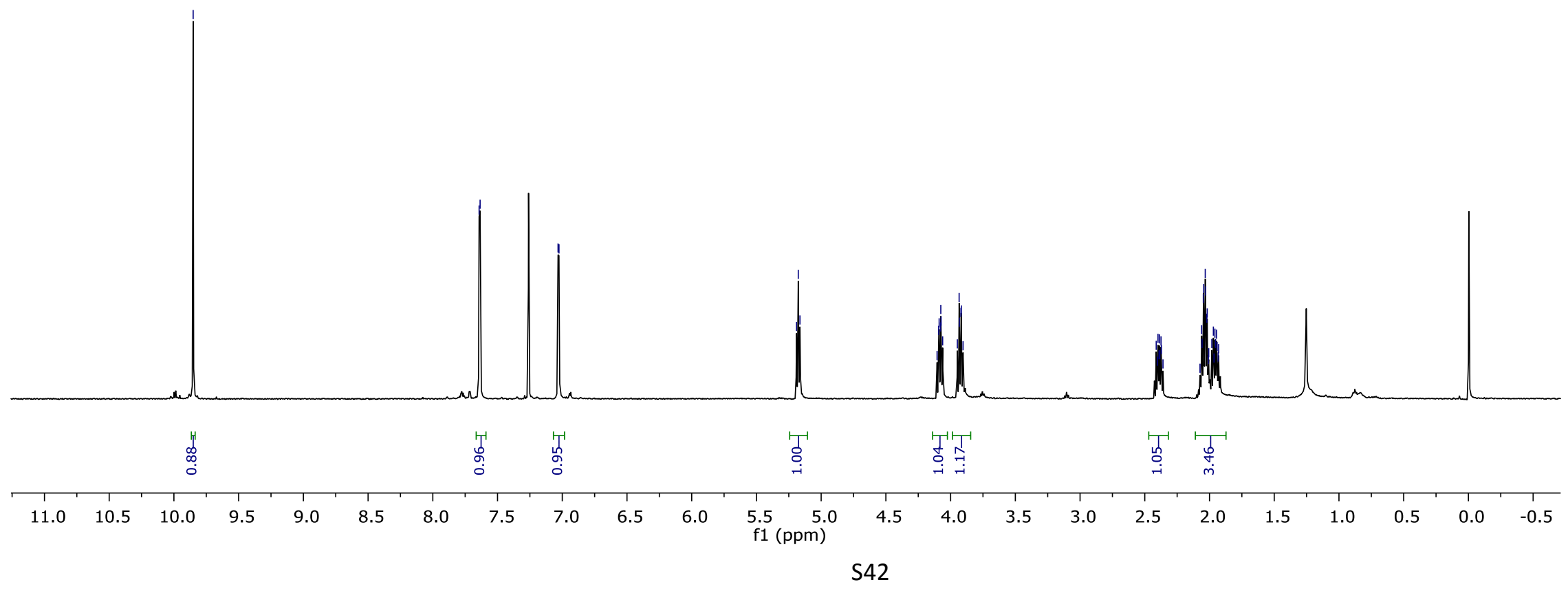


${ }^{13} \mathrm{C}$ NMR Spectrum of 5-(Tetrahydrofuran-2-yl)thiophene-2-carbaldehyde (21)<smiles>O=Cc1ccc(C2CCCO2)s1</smiles>

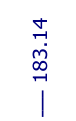

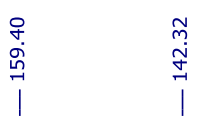

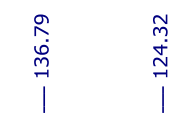

$\underset{\substack{0 \\ \infty}}{\substack{\infty \\ \infty}}$
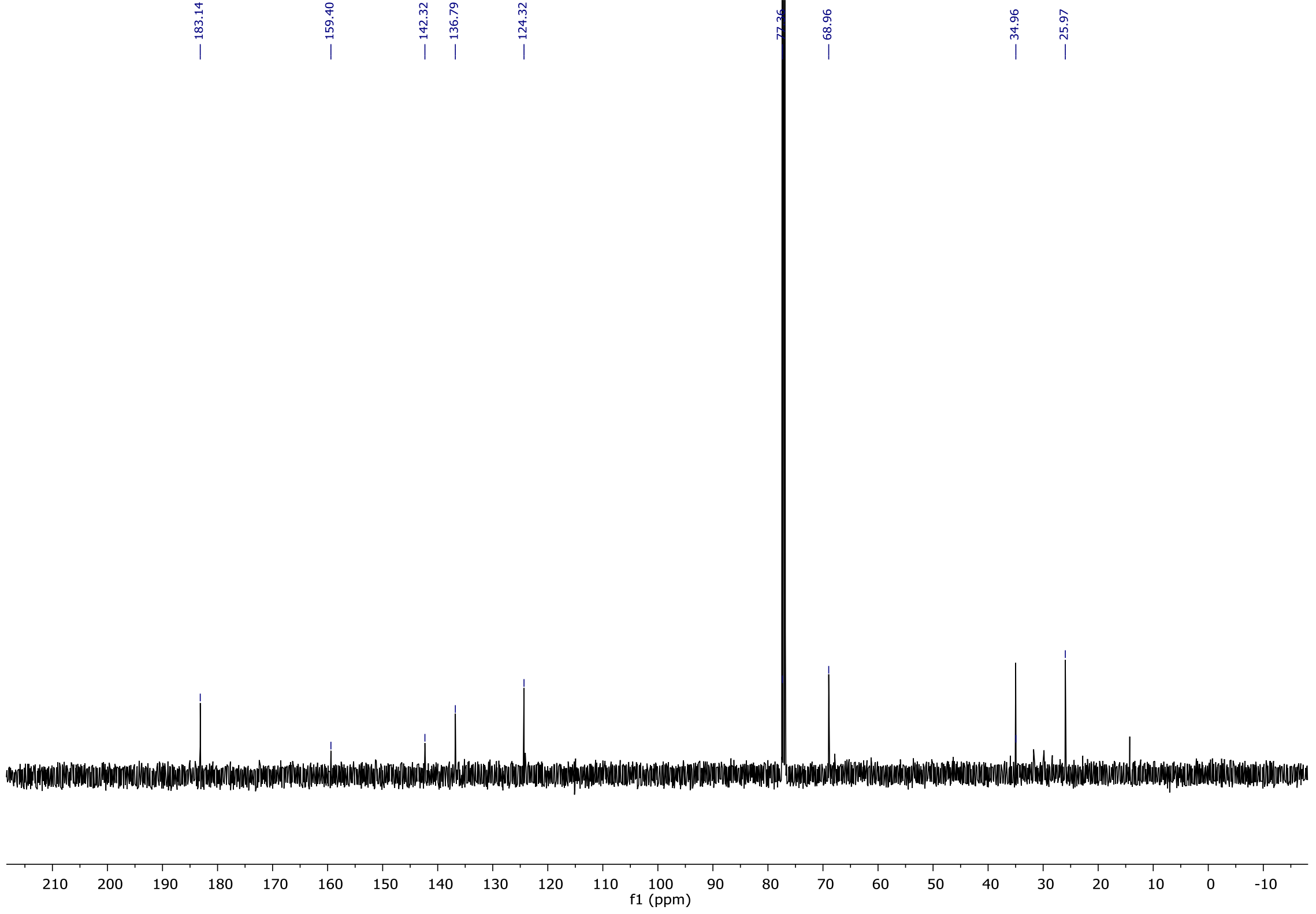

S43 
${ }^{1}$ H NMR Spectrum of 2-Fluoro-4-(tetrahydrofuran-2-yl)pyridine (22)
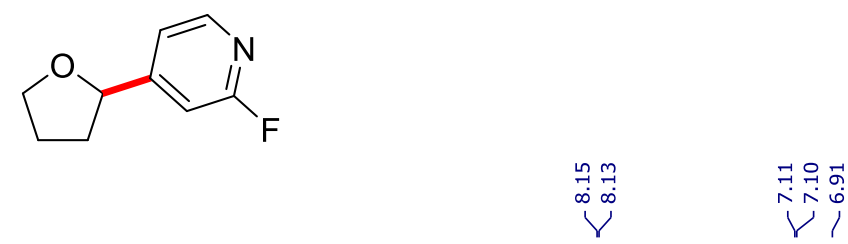

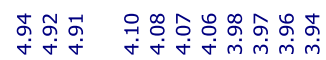

$\underbrace{+\infty}$

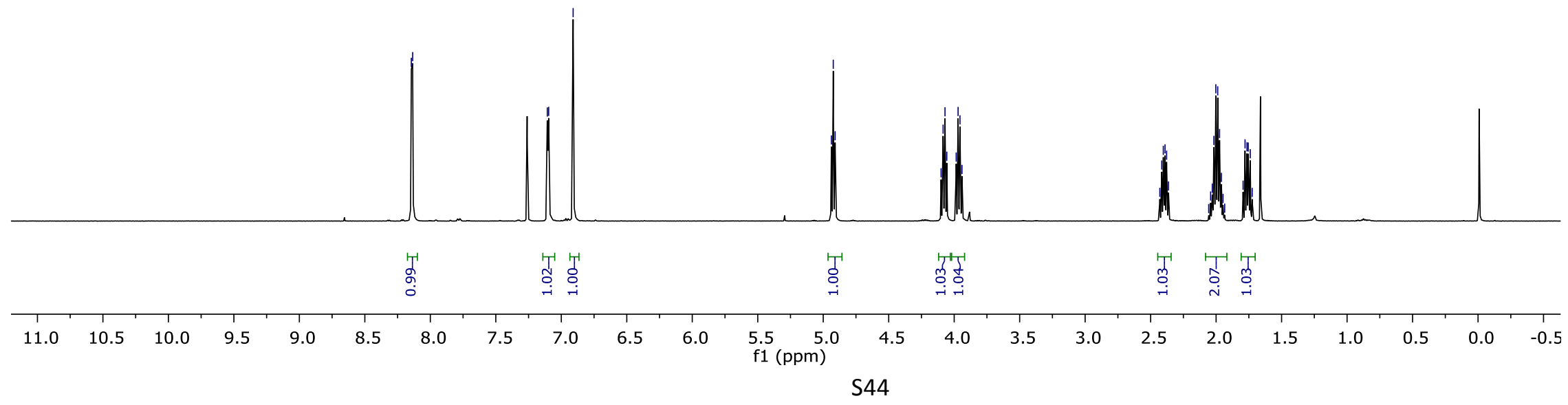


${ }^{13} \mathrm{C}$ NMR Spectrum of 2-Fluoro-4-(tetrahydrofuran-2-yl)pyridine (22)

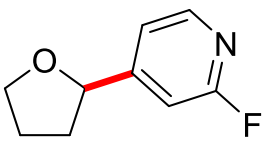

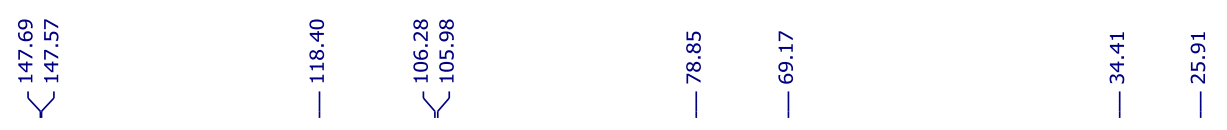

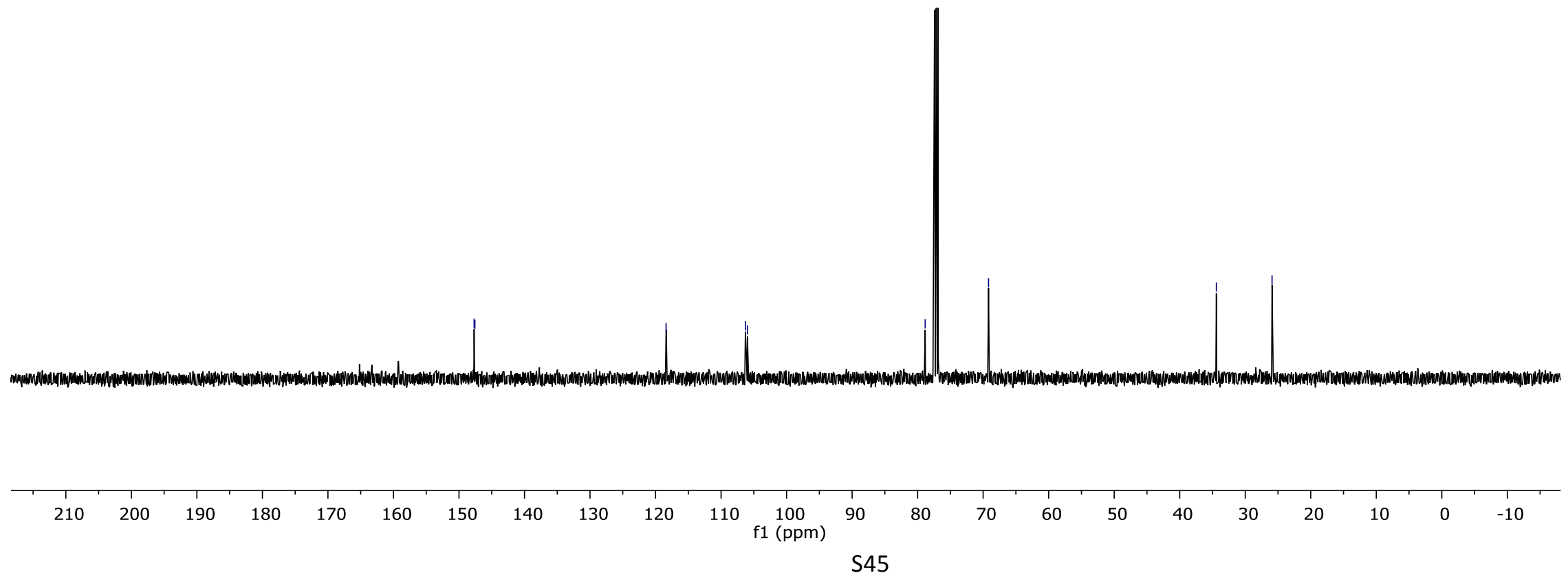


${ }^{19}$ F NMR Spectrum of 2-Fluoro-4-(tetrahydrofuran-2-yl)pyridine (22)

(N)

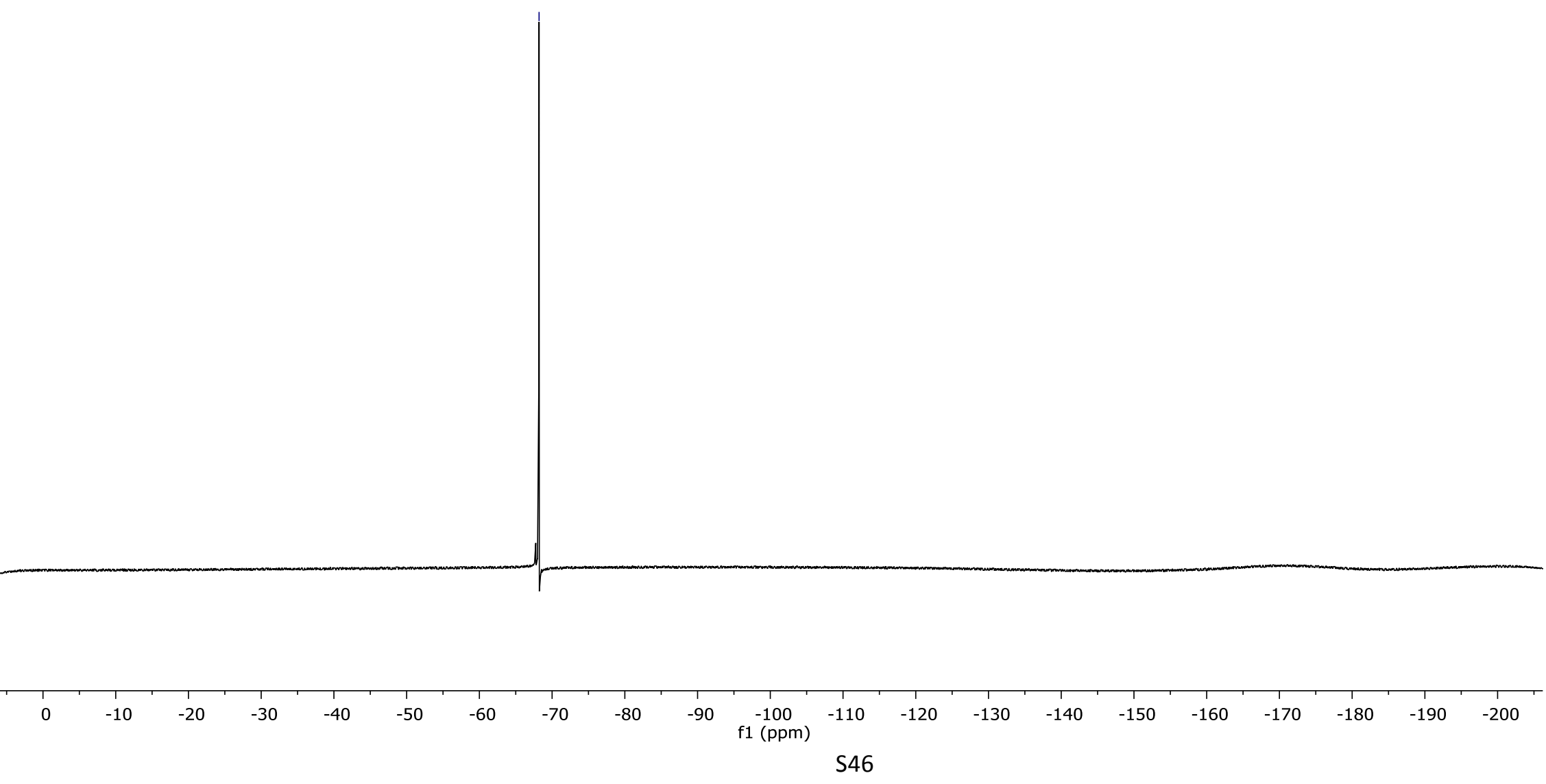


${ }^{1} \mathrm{H}$ NMR Spectrum of 3-Chloro-5(tetrahydrofuran-2-yl)pyridine (23)
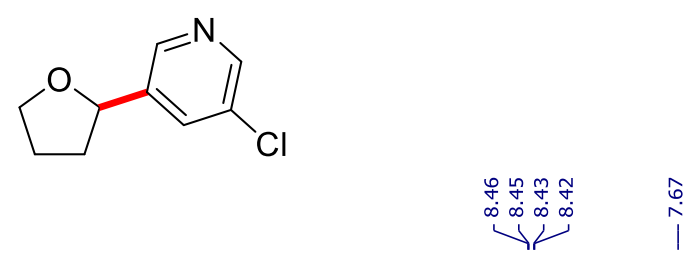

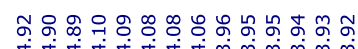

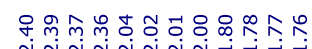
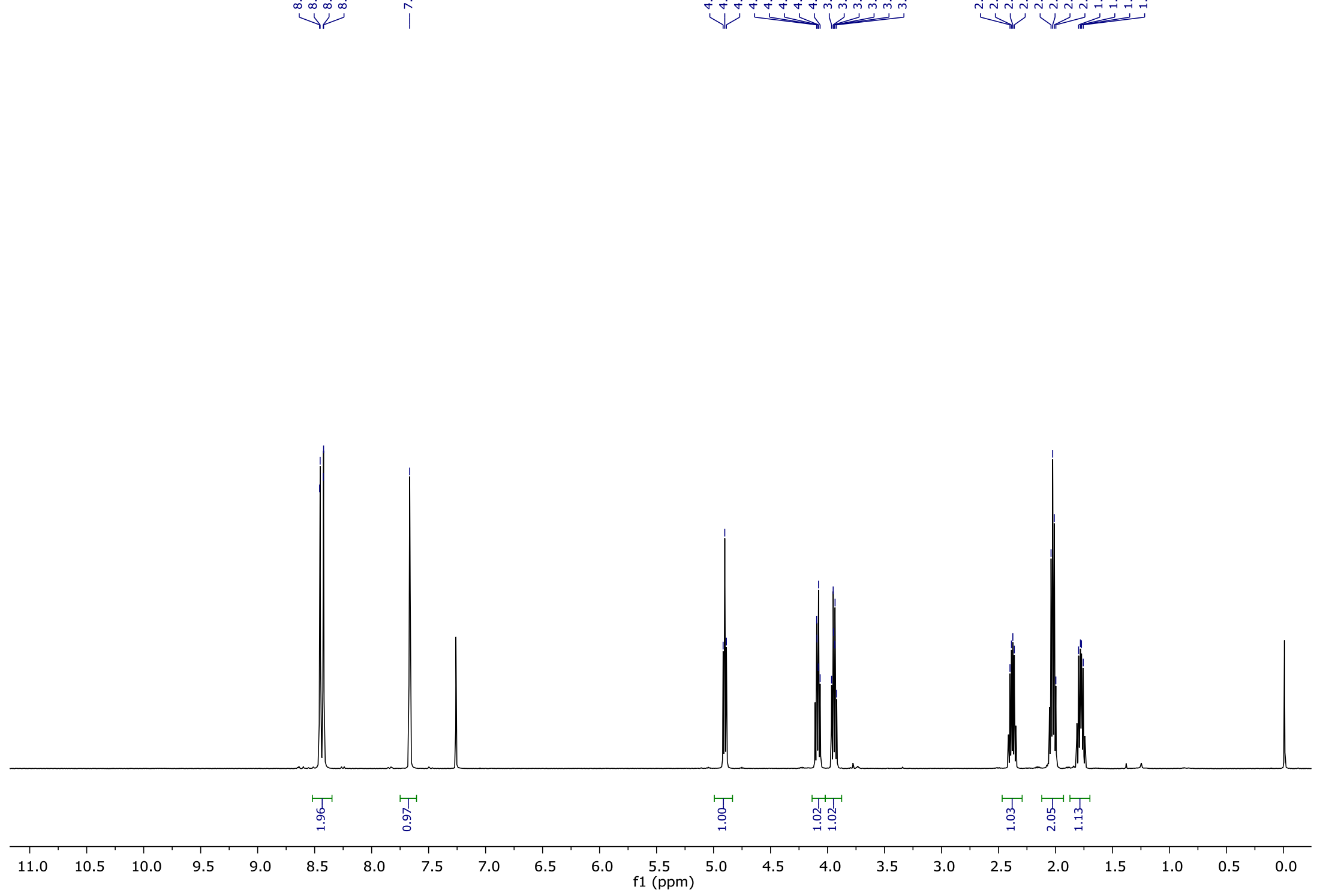
${ }^{13} \mathrm{C}$ NMR Spectrum of 3-Chloro-5(tetrahydrofuran-2-yl)pyridine (23)

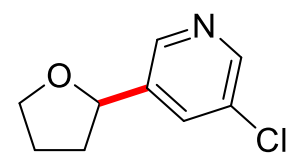

$$
\text { 要早员 }
$$

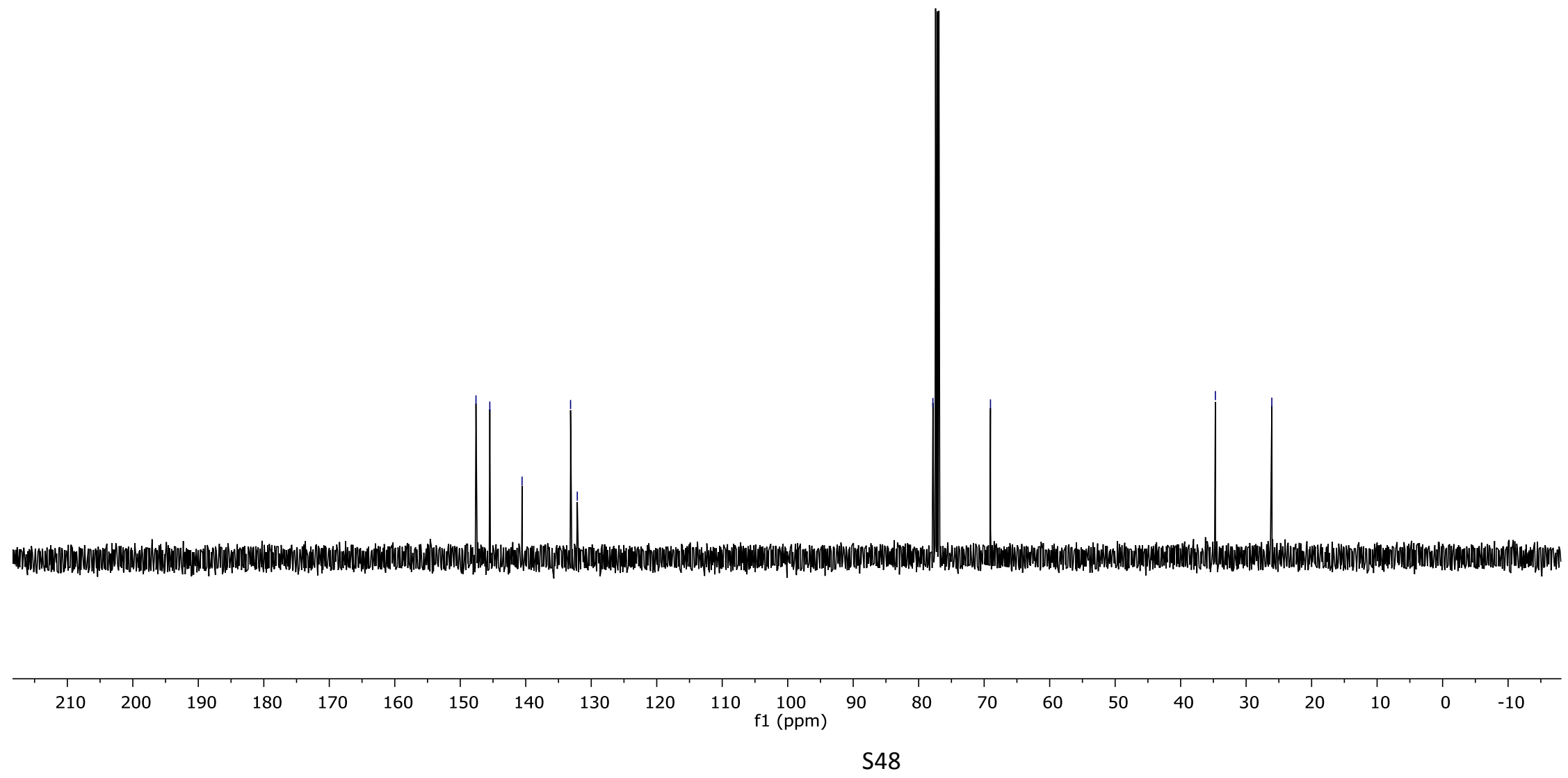


${ }^{1}$ H NMR Spectrum of 2-Phenyl-6-(tetrahydrofuran-2-yl)pyridine (24)

${ }^{O}$

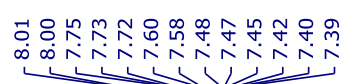

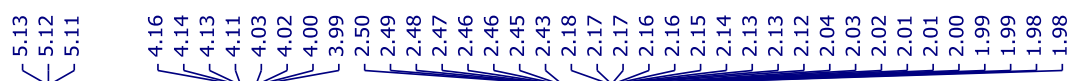

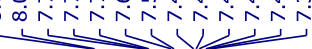

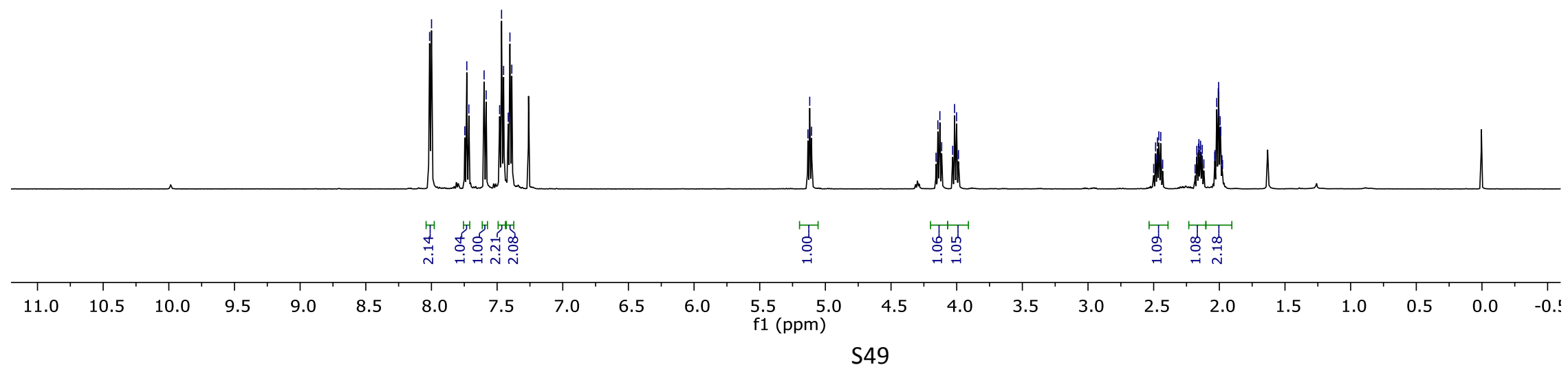


${ }^{13} \mathrm{C}$ NMR Spectrum of 2-Phenyl-6-(tetrahydrofuran-2-yl)pyridine (24)

${ }^{O}$

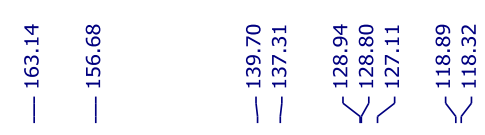

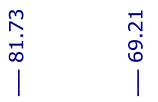

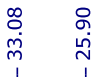

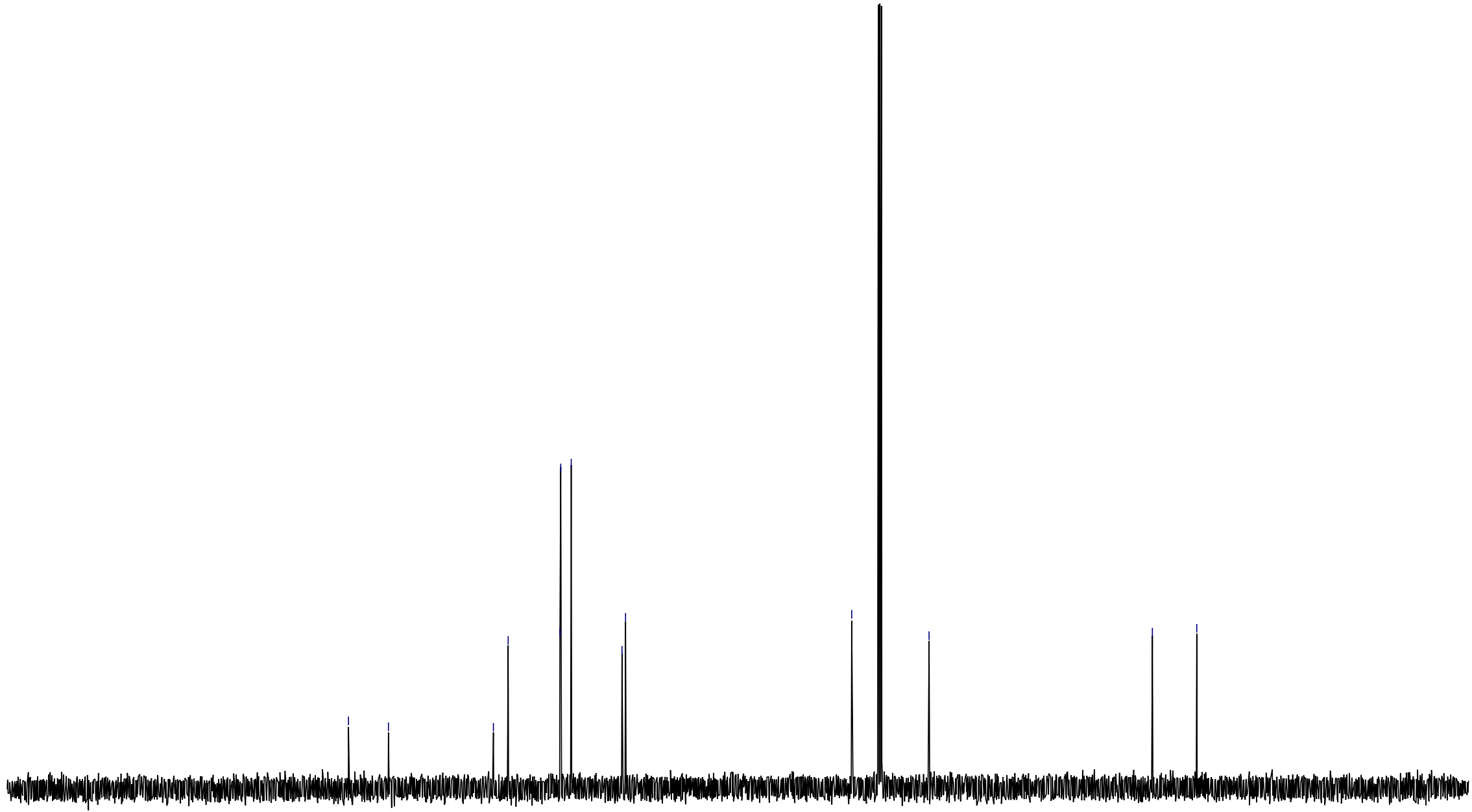

$\begin{array}{lllllllllll}210 & 200 & 190 & 180 & 170 & 160 & 150 & 140 & 130 & 120 & 110 \\ \mathrm{f} 1(\mathrm{ppm})\end{array}$

80

50




\section{References}

1. Tellis, J. C.; Primer, D. N.; Molander, G. A. Science 2014, 345, 433.

2. Oderinde, M. S.; Varela-Alvarez, A.; Aquila, B.; Robbins, D. W.; Johannes, J. W. J. Org. Chem. 2015, 80, 7642 .

3. Lie, D.; Liu, C.; Li, H.; Lei, A. Angew. Chem., Int. Ed., 2013, 52, 4453.

4. Robertson, F. J.; Wu, J. J. Am. Chem. Soc. 2012, 134, 2775.

5. Tsuji, M.; Higashiyama, K.; Yamauchi, T.; Kubo, H.; Ohmiya, S. Heterocycles 2001, 54, 1027.

6. Ueno, R.; Shirakawa, E. Org. Biomol. Chem., 2014, 12, 7469.

7. Sing, P. P.; Gudup, S.; Ambala, S.; Singh, U.; Dadhwal, S.; Singh, B.; Sawant, S. D.; Vishwakarma, R. A. Chem. Commun. 2011, 47, 5852. 